\title{
DOMINATING INDUCED MATCHINGS OF FINITE GRAPHS AND REGULARITY OF EDGE IDEALS
}

\author{
TAKAYUKI HIBI, AKIHIRO HIGASHITANI, KYOUKO KIMURA, \\ AND AKIYOSHI TSUCHIYA
}

\begin{abstract}
The regularity of an edge ideal of a finite simple graph $G$ is at least the induced matching number of $G$ and is at most the minimum matching number of $G$. If $G$ possesses a dominating induced matching, i.e., an induced matching which forms a maximal matching, then the induced matching number of $G$ is equal to the minimum matching number of $G$. In the present paper, from viewpoints of both combinatorics and commutative algebra, finite simple graphs with dominating induced matchings will be mainly studied.
\end{abstract}

\section{INTRODUCTION}

The regularity of an edge ideal of a finite simple graph has been studied by many articles including [1], 2], 6], [9], [12, [13, [15], [18, [19, [20, [22] and [23. Recall that a finite graph is simple if it possesses no loop and no multiple edge.

Let $G$ be a finite simple graph on the vertex set $[n]=\{1, \ldots, n\}$ with the edge set $E(G)$ and $S=K\left[x_{1}, \ldots, x_{n}\right]$ the polynomial ring in $n$ variables over a field $K$ with standard grading. The edge ideal of $G$ is the ideal $I(G) \subset S$ which is generated by those squarefree quadratic monomials $x_{i} x_{j}$ with $\{i, j\} \in E(G)$. Following the previous paper [10, we continue our research on the relation between the regularity $\operatorname{reg}(S / I(G))$ of the quotient ring $S / I(G)$ and the matching number, the minimum matching number together with the induced matching number of $G$.

A matching of $G$ is a subset $\mathcal{M} \subset E(G)$ such that, for $e$ and $e^{\prime}$ belonging to $\mathcal{M}$ with $e \neq e^{\prime}$, one has $e \cap e^{\prime}=\emptyset$. A maximal matching of $G$ is a matching $\mathcal{M}$ of $G$ for which $\mathcal{M} \cup\{e\}$ cannot be a matching of $G$ for all $e \in E(G) \backslash \mathcal{M}$. An induced matching is a matching $\mathcal{M}$ of $G$ such that, for $e$ and $e^{\prime}$ belonging to $\mathcal{M}$ with $e \neq e^{\prime}$, there is no edge $f \in E(G)$ with $e \cap f \neq \emptyset$ and $e^{\prime} \cap f \neq \emptyset$. The matching number of $G$, denoted by match $(G)$, is the maximum cardinality of the matchings of $G$ and the minimum matching number of $G$, denoted by min-match $(G)$, is the minimum cardinality of the maximal matchings of $G$. Furthermore, the induced matching number of $G$, denoted by ind-match $(G)$, is the maximum cardinality of the induced matching of $G$.

The basic inequalities, due to [12] and [22], among the above three invariants together with $\operatorname{reg}(S / I(G))$ are

$$
\text { ind-match }(G) \leq \operatorname{reg} S / I(G) \leq \min -\operatorname{match}(G) \leq \operatorname{match}(G) .
$$

2010 Mathematics Subject Classification. Primary 05E40; Secondly 05C69, 05C70.

Key words and phrases. edge ideal, dominating induced matching, regularity, unmixed graph, vertex decomposable graph. 
In addition, one can easily prove the inequality

$$
\operatorname{match}(G) \leq 2 \text { min-match }(G),
$$

see Proposition 1.1. Naturally, one question arises: Given integers $p, c, q, r$ satisfying

$$
0<p \leq c \leq q \leq r \leq 2 q,
$$

we can ask if there exists a finite simple graph $G$ for which

$$
\text { ind-match }(G)=p, \operatorname{reg} S / I(G)=c, \min -\operatorname{match}(G)=q, \operatorname{match}(G)=r .
$$

In Section 1, this question and its related problems will be studied.

Cameron and Walker [4] succeeded in characterizing a finite simple graph $G$ with ind-match $(G)=\operatorname{match}(G)$. For example, if $G$ is a star or a star triangle, then one has ind-match $(G)=\operatorname{match}(G)$. We say that a finite connected simple graph $G$ is a Cameron-Walker graph if ind-match $(G)=\operatorname{match}(G)$ and if $G$ is neither a star nor a star triangle. Thus in particular for a Cameron-Walker graph $G$, one has

$$
\text { ind-match }(G)=\operatorname{reg} S / I(G)=\min -\operatorname{match}(G)=\operatorname{match}(G) \text {. }
$$

From a viewpoint of commutative algebra, the study on Cameron-Walker graphs is done in 10. In Section 2, we treat some classes of finite simple graphs which contain Cameron-Walker graphs as a subclass and investigate these combinatorial properties.

A dominating induced matching of $G$ is an induced matching which also forms a maximal matching of $G$. Every Cameron-Walker graph possesses a dominating induced matching. Clearly a finite simple graph $G$ with a dominating induced matching satisfies the equalities

$$
\text { ind-match }(G)=\operatorname{reg} S / I(G)=\min -\operatorname{match}(G) .
$$

However, there is a finite simple graph $G$ which possesses no dominating induced matching, but satisfies the equality ind-match $(G)=\min -\operatorname{match}(G)$. A characterization of finite simple graphs possessing dominating induced matchings is easy, see Proposition 2.1.

Our first work is to find a characterization of finite simple graphs $G$ satisfying ind-match $(G)=\min -\operatorname{match}(G)$ (Theorem 2.3).

Recall that a vertex cover of a finite simple graph $G$ on $[n]$ is a subset $C \subset[n]$ for which $C \cap e \neq \emptyset$ for all $e \in E(G)$. A minimal vertex cover of $G$ is a vertex cover $C$ of $G$ for which no proper subset of $C$ can be a vertex cover of $G$. A finite simple graph $G$ is called unmixed if all minimal vertex covers have the same cardinality. Our second work is to characterize unmixed graphs with dominating induced matchings (Theorem 2.4).

Finally, in Section 3, the algebraic study of finite simple graphs with dominating induced matchings will be discussed. In [10] it is shown that every Cameron-Walker graph is vertex decomposable, hence sequentially Cohen-Macaulay. However, there is a finite simple graph $G$ with a dominating induced matching such that $G$ is not sequentially Cohen-Macaulay. We cannot escape from the temptation to find a characterization of vertex decomposable graphs with dominating induced matchings. However, to find a complete characterization seems to be rather difficult. We try to find a class $\mathcal{A}$ of vertex decomposable graphs with dominating induced matchings such that $\mathcal{A}$ contains all Cameron-Walker graphs. In addition, various examples will be supplied. 


\section{MATChing Number, INDUCED MATCHING NUMBER, AND REGUlaRity}

Let $G$ be a finite simple graph. A matching of $G$ is a subset $\mathcal{M} \subset E(G)$ such that $e \cap e^{\prime}=\emptyset$ for all $e, e^{\prime} \in \mathcal{M}$ with $e \neq e^{\prime}$. We denote by $\operatorname{match}(G)$ (resp. min-match $(G)$ ), the maximum (resp. minimum) cardinality among maximal matchings of $G$. Two edges $e, e^{\prime} \in E(G)$ are said to be 3 -disjoint if $e \cap e^{\prime}=\emptyset$ and there is no edge $f \in E(G)$ with $e \cap f \neq \emptyset$ and $e^{\prime} \cap f \neq \emptyset$. An induced matching is a set of edges which are pairwise 3-disjoint. We denote by ind-match $(G)$, the maximum cardinality among induced matchings of $G$. By Katzman [12] and Woodroofe [22, we have

$$
\text { ind-match }(G) \leq \operatorname{reg} S / I(G) \leq \min -\operatorname{match}(G) \leq \operatorname{match}(G)
$$

In this section, we investigate the problem to construct a finite simple connected graph with given values of these 4 invariants.

We first note the relation between $\operatorname{match}(G)$ and $\min -\operatorname{match}(G)$.

Proposition 1.1. Let $G$ be a finite simple graph. Then $\operatorname{match}(G) \leq 2$ min-match $(G)$.

Proof. Let $\left\{u_{i}, v_{i}\right\}, i=1,2, \ldots, q$ be edges of $G$ which form a maximal matching with $q=\min -\operatorname{match}(G)$. Let $e$ be an edge in $G$. Then $e$ contains at least one vertex of $2 q$ vertices $u_{1}, v_{1}, \ldots, u_{q}, v_{q}$. Therefore there is no matching which consists of $2 q+1$ edges.

Then the following problem naturally occurs:

Problem 1.2. Let $p, c, q, r$ be integers satisfying

$$
0<p \leq c \leq q \leq r \leq 2 q
$$

Construct a finite simple connected graph $G$ satisfying

$$
\text { ind-match }(G)=p, \quad \operatorname{reg} S / I(G)=c, \quad \min -\operatorname{match}(G)=q, \quad \operatorname{match}(G)=r .
$$

When we ignore the condition for the regularity, we can do. The following result might be known, however we give a proof of it for the sake of completeness.

Theorem 1.3. For arbitrary integers $p, q, r$ with $0<p \leq q \leq r \leq 2 q$, there exists a finite simple connected graph $G$ which satisfies

$$
\operatorname{ind}-\operatorname{match}(G)=p, \quad \min -\operatorname{match}(G)=q, \quad \operatorname{match}(G)=r \text {. }
$$

Proof. Let $a, b, m, n$ be non-negative integers with $m \leq n$ and $1 \leq n$. Let us consider the following graph $G_{a, b, m, n}$ : 


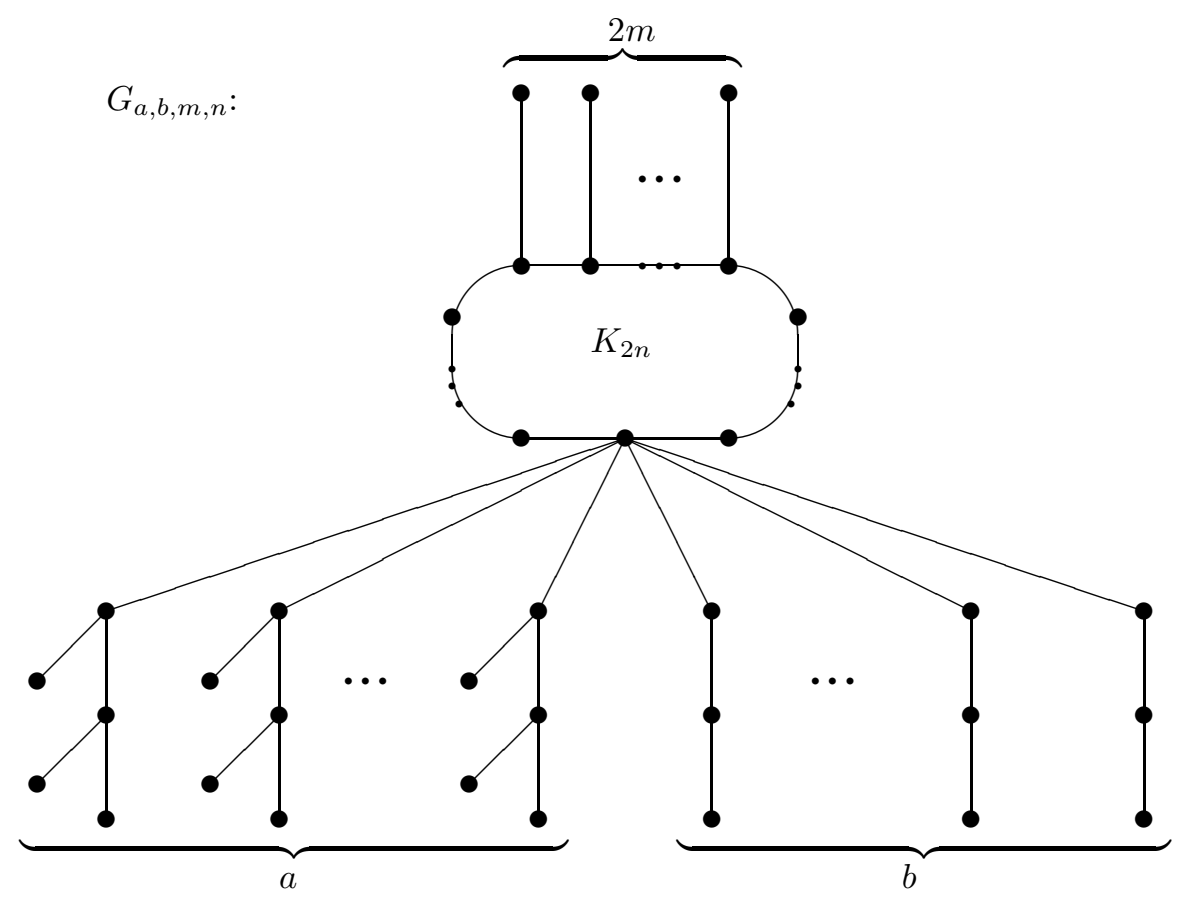

Then $G_{a, b, m, n}$ satisfies

$$
\begin{aligned}
\operatorname{ind}-\operatorname{match}\left(G_{a, b, m, n}\right) & =a+b+1, \\
\min -\operatorname{match}\left(G_{a, b, m, n}\right) & =a+b+n, \\
\operatorname{match}\left(G_{a, b, m, n}\right) & =2 a+b+(n-m)+2 m=2 a+b+n+m .
\end{aligned}
$$

Therefore we obtain a desired graph $G$ if we can choose $a, b, n, m$ satisfying

$$
\begin{aligned}
a+b+1 & =p, \\
a+b+n & =q, \\
2 a+b+n+m & =r .
\end{aligned}
$$

Indeed, we can choose such $a, b, m, n$. First, by $a+b=p-1$, we take $n=$ $q-(a+b)=q-p+1>0$. Then

$$
r=2 a+b+n+m=a+(p-1)+(q-p+1)+m,
$$

and we have $a+m=r-q$. Note that $a+b=p-1$ and $a+m=r-q$.

Case 1: $r-q \leq q-p+1$. We can take $a=0, b=p-1$, and $m=r-q$. (Then $m \leq n$.

Case 2: $r-q>q-p+1$. We set $m=q-p+1$ and $a=r-2 q+p-1(>0)$. Then $m=n$ and $b=(p-1)-(r-2 q+p-1)=2 q-r \geq 0$. The last inequality follows from the condition $r \leq 2 q$.

Also, the difference between the regularity and the induced matching number as well as the difference between the minimum matching number and the regularity can be arbitrary large.

Recall that the regularity of a (standard graded) $S$-module $M$ is defined by

$$
\operatorname{reg}(M)=\max \left\{j-i: \beta_{i j}(M) \neq 0\right\},
$$


where $\beta_{i j}(M):=\operatorname{dim}_{K}\left[\operatorname{Tor}_{i}^{S}(K, M)\right]_{j}$, the $i j$ th Betti number of $M$.

Theorem 1.4. For arbitrary non-negative integers $a, b$, there exists a finite simple connected graph $G$ satisfying

$$
\begin{aligned}
\operatorname{ind}-\operatorname{match}(G) & =\operatorname{reg}(S / I(G))-a, \\
\min -\operatorname{match}(G) & =\operatorname{reg}(S / I(G))+b .
\end{aligned}
$$

Remark 1.5. Although for a given integer $c$, there exists a simple connected graph $G$ with $\operatorname{reg} S / I(G)=c$ (for example, the cycle of length $3 c-1$ is such a graph), we do not know whether there exists a finite simple connected graph $G$ satisfying $\operatorname{reg} S / I(G)=c$ together with (1.2) for given integers $a, b, c$.

Let $G$ be a finite simple graph on $V$. When we identify the vertices of $G$ with the variables of the underlying polynomial ring $S$ of the edge ideal $I(G)$, we denote $S=K[V]$. Theorem 1.4 immediately follows by the following lemma.

Lemma 1.6. Let $a, b$ be non-negative integers. Let $G_{a, b}$ be the graph consisting of a complete bipartite graph $K_{1, a+b+1}$ with the bipartition $\{x\} \sqcup\left\{y_{1}, \ldots, y_{a+b+1}\right\}$ and 5 cycles attaching to each $y_{1}, \ldots, y_{a+1}$ and 4 -cycles attaching to each $y_{a+2}, \ldots, y_{a+b+1}$. $W e$ denote by $V_{a, b}$, the vertex set of $G_{a, b}$. Then

$$
\begin{aligned}
\operatorname{ind}-\operatorname{match}\left(G_{a, b}\right) & =a+b+2, \\
\min -\operatorname{match}\left(G_{a, b}\right) & =2 a+2 b+2, \\
\operatorname{match}\left(G_{a, b}\right) & =2 a+2 b+3, \\
\operatorname{reg}\left(K\left[V_{a, b}\right] / I\left(G_{a, b}\right)\right) & =2 a+b+2 .
\end{aligned}
$$

In order to prove Lemma 1.6 we use the following two results.

Lemma 1.7 (Woodroofe 22, Corollary 10]). If a graph $G$ has an induced subgraph $H$ which consists of disjoint union of $m$ edges and cycles $C_{3 i_{1}+2}, \ldots, C_{3 i_{n}+2}$, then $\operatorname{reg} S / I(G) \geq m+n+\sum_{j=1}^{n} i_{j}$.

Lemma 1.8 (Kalai and Meshulam [11, Theorem 1.2]). Let $I_{1}, \ldots, I_{s}$ be squarefree monomial ideals of $S$. Then

$$
\operatorname{reg} S /\left(I_{1}+\cdots+I_{s}\right) \leq \sum_{j=1}^{s} \operatorname{reg} S / I_{j}
$$

Proof of Lemma 1.6. We first compute ind-match $\left(G_{a, b}\right)$. Note that we cannot choose 2 edges which are 3-disjoint in $G_{a, b}$ from each 4-cycle or each 5-cycle. The same is true for $K_{1, a+b+1}$. Therefore ind-match $\left(G_{a, b}\right) \leq a+b+2$. Indeed, there exist $a+b+2$ edges of $G_{a, b}$ which form an induced matching of $G_{a, b}$ : we choose an edge which does not contain $y_{i}$ from each 4-cycle, the edge which is 3-disjoint with $\left\{x, y_{i}\right\}$ from each 5-cycle, and $\left\{x, y_{1}\right\}$.

We next compute reg $K\left[V_{a, b}\right] / I\left(G_{a, b}\right)$. Take an edge from each 4-cycle. Then the graph which consists of these $b$ edges and $a+1$ copies of 5-cycles is an induced subgraph of $G_{a, b}$. Therefore by Lemma 1.7, we have

$$
\operatorname{reg}\left(K\left[V_{a, b}\right] / I\left(G_{a, b}\right)\right) \geq b+2(a+1) .
$$

In order to prove the opposite inequality, we define subgraphs $G_{1}, \ldots, G_{a+b+1}$ of $G_{a, b}$. For $1 \leq i \leq a+1$, let $G_{i}$ be the subgraph of $G_{a, b}$ consisting of the 5-cycle containing $y_{i}$ and $\left\{x, y_{i}\right\}$. Also for $a+2 \leq i \leq a+b+1$, let $G_{i}$ be the subgraph of $G_{a, b}$ 
consisting of the 4-cycle containing $y_{i}$ and $\left\{x, y_{i}\right\}$. Then $E\left(G_{a, b}\right)=\bigcup_{i=1}^{a+b+1} E\left(G_{i}\right)$. Since

$$
\operatorname{reg}\left(K\left[V_{a, b}\right] / I\left(G_{i}\right) K\left[V_{a, b}\right]\right)= \begin{cases}2, & 1 \leq i \leq a+1, \\ 1, & a+2 \leq i \leq a+b+1,\end{cases}
$$

we have $\operatorname{reg}\left(K\left[V_{a, b}\right] / I\left(G_{a, b}\right)\right) \leq 2(a+1)+b$ by Lemma 1.8

Therefore $\operatorname{reg}\left(K\left[V_{a, b}\right] / I\left(G_{a, b}\right)\right)=b+2(a+1)$ holds.

Finally we compute min-match $\left(G_{a, b}\right)$ and $\operatorname{match}\left(G_{a, b}\right)$. Note that

$$
\begin{aligned}
& \min -\operatorname{match}\left(C_{4}\right)=\operatorname{match}\left(C_{4}\right)=2, \\
& \min -\operatorname{match}\left(C_{5}\right)=\operatorname{match}\left(C_{5}\right)=2, \\
& \min -\operatorname{match}\left(K_{1, a+b+1}\right)=\operatorname{match}\left(K_{1, a+b+1}\right)=1 .
\end{aligned}
$$

Let $\mathcal{M}$ be a maximal matching of $G$. If $\left\{x, y_{i}\right\} \notin \mathcal{M}$ for $i=1, \ldots, a+b+1$, then we have $\# \mathcal{M}=2(a+1)+2 b$. If $\left\{x, y_{i}\right\} \in \mathcal{M}$ for some $1 \leq i \leq a+1$, then the cardinality of $\mathcal{M}$ is either $1+2(a+1)+2 b$ or $1+1+2 a+2 b=2(a+1)+2 b$. If $\left\{x, y_{i}\right\} \in \mathcal{M}$ for some $a+2 \leq i \leq a+b+1$, then we have $\# \mathcal{M}=2(a+1)+2 b$. Therefore we have the desired assertions.

Now we return to the first inequalities (1.1). There are the following 8 cases:

(i) $\operatorname{ind}-\operatorname{match}(G)=\operatorname{reg} S / I(G)=\min -\operatorname{match}(G)=\operatorname{match}(G)$.

(ii) ind-match $(G)=\operatorname{reg} S / I(G)=\min -\operatorname{match}(G)<\operatorname{match}(G)$.

(iii) $\operatorname{ind}-\operatorname{match}(G)=\operatorname{reg} S / I(G)<\min -\operatorname{match}(G)=\operatorname{match}(G)$.

(iv) ind-match $(G)=\operatorname{reg} S / I(G)<\min -\operatorname{match}(G)<\operatorname{match}(G)$.

(v) $\operatorname{ind}-\operatorname{match}(G)<\operatorname{reg} S / I(G)=\min -\operatorname{match}(G)=\operatorname{match}(G)$.

(vi) ind-match $(G)<\operatorname{reg} S / I(G)=\min -\operatorname{match}(G)<\operatorname{match}(G)$.

(vii) ind-match $(G)<\operatorname{reg} S / I(G)<\min -\operatorname{match}(G)=\operatorname{match}(G)$.

(viii) ind-match $(G)<\operatorname{reg} S / I(G)<\min -\operatorname{match}(G)<\operatorname{match}(G)$.

For each case, is there a finite simple connected graph $G$ satisfying the inequalities? The following theorem is an answer to the question.

Theorem 1.9. There exists a finite simple connected graph $G$ satisfying the inequalities. In particular, we can construct an infinite family of finite simple connected graphs satisfying each inequalities except for the case (v).

A graph $G$ is called chordal if any cycle in $G$ of length more than 3 has a chord. Hà and Van Tuyl [9] proved that $\operatorname{reg} S / I(G)=\operatorname{ind}-\operatorname{match}(G)$ holds for a chordal graph $G$.

Proof of Theorem 1.9. Case (i): The Cameron-Walker graphs [10] are just such graphs $G$.

Case (ii): The path graph $P_{6 n}$ with $6 n$ vertices $(n \geq 1)$ satisfies the inequalities. Indeed, ind-match $\left(P_{6 n}\right)=\min -\operatorname{match}\left(P_{6 n}\right)=2 n$ and $\operatorname{match}\left(P_{6 n}\right)=3 n$. (Note that $P_{6 n}$ has a dominating induced matching; see Section 2 .)

Case (iii): The complete graph $K_{n}$ with $n$ vertices $(n \geq 4)$ satisfies the inequalities. Indeed, ind-match $\left(K_{n}\right)=1$ and min-match $\left(K_{n}\right)=\operatorname{match}\left(K_{n}\right)=\lfloor n / 2\rfloor \geq 2$. Also, since $K_{n}$ is a chordal graph, it follows that ind-match $\left(K_{n}\right)=\operatorname{reg}\left(S / I\left(K_{n}\right)\right)$ by 9 .

Case (iv): The fully whiskered graph $W\left(K_{n}\right)$ of the complete graph $K_{n}(n \geq$ 3 ) satisfies the inequalities. Here $W\left(K_{n}\right)$ is defined as follows: let $x_{1}, \ldots, x_{n}$ be vertices of $K_{n}$ and let $y_{1}, \ldots, y_{n}$ be new vertices. Then $W\left(K_{n}\right)$ is the graph on $\left\{x_{1}, \ldots, x_{n}, y_{1}, \ldots, y_{n}\right\}$ whose edge set is $E\left(K_{n}\right) \cup\left\{\left\{x_{i}, y_{i}\right\}: i=1,2, \ldots, n\right\}$. Note 
that $W\left(K_{n}\right)$ is also a chordal graph. Thus ind-match $(G)=\operatorname{reg} S / I(G)$ holds. Since ind-match $(G)=1$, min-match $(G)=\lceil n / 2\rceil$ and $\operatorname{match}(G)=n, G$ satisfies the desired inequalities.

Case (v): The 5-cycle $C_{5}$ satisfies the desired inequalities. Indeed, ind-match $\left(C_{5}\right)=$ 1 and $\operatorname{reg} S / I\left(C_{5}\right)=\min -\operatorname{match}\left(C_{5}\right)=\operatorname{match}\left(C_{5}\right)=2$.

Cases (vi) and (viii): Consider the graph $G_{a, b}$ on Lemma 1.6. If $a \neq 0$ and $b=0$, then the graph satisfies the inequalities (vi). If $a \neq 0$ and $b \neq 0$, then the graph satisfies the inequalities (viii).

Cases (vii): For an integer $k \geq 2$, let $H_{k}$ be the graph on

$$
V_{k}:=\left\{u, v, x_{1}, x_{2}, \ldots, x_{k}, y_{1}, y_{2}, \ldots, y_{k}, z_{11}, z_{12}, z_{21}, z_{22}, \ldots, z_{k 1}, z_{k 2}\right\}
$$

with the following edges:

$$
\begin{aligned}
\mathfrak{e}_{i} & :=\left\{u, x_{i}\right\}, \quad \mathfrak{f}_{i}:=\left\{v, y_{i}\right\}, \\
\mathfrak{g}_{i 1} & :=\left\{x_{i}, z_{i 1}\right\}, \mathfrak{g}_{i 2}:=\left\{x_{i}, z_{i 2}\right\}, \quad i=1,2, \ldots, k ; \\
\mathfrak{h}_{i 1} & :=\left\{y_{i}, z_{i 1}\right\}, \mathfrak{h}_{i 2}:=\left\{y_{i}, z_{i 2}\right\},
\end{aligned}
$$

see Figure 1

Then we see from Lemma 1.10 below that $H_{k}$ satisfies the inequalities (vii).

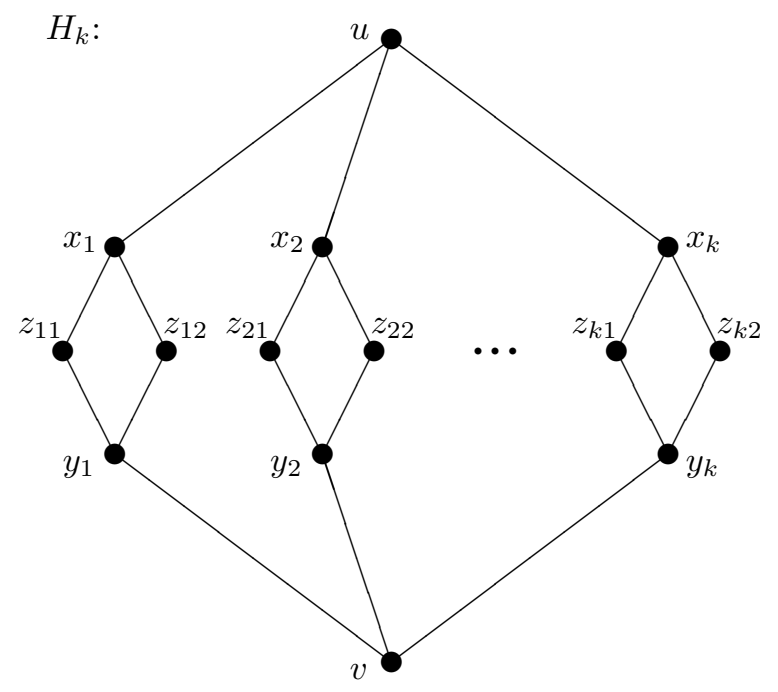

FiguRE 1. The graph $H_{k}$

Lemma 1.10. The graph $H_{k}(k \geq 2)$ in the proof of Theorem 1.9] satisfies

$$
\begin{aligned}
\operatorname{ind}-\operatorname{match}\left(H_{k}\right) & =k, \\
\min -\operatorname{match}\left(H_{k}\right) & =\operatorname{match}\left(H_{k}\right)=2 k, \\
\operatorname{reg}\left(K\left[V_{k}\right] / I\left(H_{k}\right)\right) & =k+1 .
\end{aligned}
$$


In order to prove Lemma 1.10 we use a Lyubeznik resolution ([17]), which is a subcomplex of the Taylor resolution.

Let $I$ be a monomial ideal of $S$ and $m_{1}, \ldots, m_{\mu}$ the minimal monomial generators of $I$. The free basis $e_{i_{1} \cdots i_{s}}$ of the Taylor resolution is said to be $L$-admissible if $\operatorname{lcm}\left(m_{i_{t}}, \ldots, m_{i_{s}}\right)$ is not divisible by $m_{q}$ for all $1 \leq t<s$ and for all $q<i_{t}$. We will denote an $L$-admissible symbol $e_{i_{1} \cdots i_{s}}$ by $\left[m_{i_{1}}, \ldots, m_{i_{s}}\right]$. The degree of an $L$-admissible symbol $\left[m_{i_{1}}, \ldots, m_{i_{s}}\right]$ is defined by the degree of $\operatorname{lcm}\left(m_{i_{1}}, \ldots, m_{i_{s}}\right)$. (Recall that we consider the standard grading on the polynomial ring $S$.) An $L$ admissible symbol $\left[m_{i_{1}}, \ldots, m_{i_{s}}\right]$ is said to be maximal if there is no $L$-admissible symbol $\left[m_{j_{1}}, \ldots, m_{j_{t}}\right]$ such that $\left\{i_{1}, \ldots, i_{s}\right\} \subsetneq\left\{j_{1}, \ldots, j_{t}\right\}$. A Lyubeznik resolution $\left(\mathcal{L}_{\bullet}, d_{\bullet}\right)$ of $I$ (with respect to the above order of the minimal monomial generators) is the subcomplex of the Taylor resolution generated by all $L$-admissible symbols, which is also a free resolution of $S / I$.

Proof of Lemma 1.10. We first compute ind-match $\left(H_{k}\right)$. Let $\mathcal{M}$ be a maximal induced matching of $H_{k}$, i.e., $\mathcal{M}$ is an induced matching of $H_{k}$ and there is no induced matching which properly contains $\mathcal{M}$. Suppose that $\left\{u, x_{1}\right\} \in \mathcal{M}$. If $\left\{v, y_{j}\right\} \in \mathcal{M}(j=1,2, \ldots, k)$, then each of the rest edges is not 3-disjoint with at least one of $\left\{u, x_{1}\right\},\left\{v, y_{j}\right\}$. Hence $\# \mathcal{M}=2$. If $\left\{v, y_{j}\right\} \notin \mathcal{M}$ for all $j$, then $\mathcal{M}$ contains exactly one of $\left\{y_{\ell}, z_{\ell 1}\right\},\left\{y_{\ell}, z_{\ell 2}\right\}$ for each $\ell=2,3, \ldots, k$. Therefore $\# \mathcal{M}=1+(k-1)=k$. When $\left\{u, x_{j}\right\},\left\{v, y_{j}\right\} \notin \mathcal{M}$ for all $j$, exactly one of $\left\{x_{j}, z_{j 1}\right\},\left\{x_{j}, z_{j 2}\right\},\left\{y_{j}, z_{j 1}\right\},\left\{y_{j}, z_{j 2}\right\}$ belongs to $\mathcal{M}$. Thus $\# \mathcal{M}=k$. Therefore we have ind-match $\left(H_{k}\right)=k$.

Let $\mathcal{M}^{\prime}$ be a maximal matching of $H_{k}$. We show that $\# \mathcal{M}^{\prime}=2 k$. Suppose that $\left\{u, x_{1}\right\} \in \mathcal{M}^{\prime}$. If $\left\{v, y_{1}\right\} \in \mathcal{M}^{\prime}$, then $\mathcal{M}^{\prime} \backslash\left\{\left\{u, x_{1}\right\},\left\{v, y_{1}\right\}\right\}$ is a maximal matching of $k-1$ copies of the 4 -cycle whose cardinality is $2(k-1)$. Hence $\# \mathcal{M}^{\prime}=2 k$. If $\left\{v, y_{j}\right\} \in \mathcal{M}^{\prime}$ for $j \neq 1$, then $\mathcal{M}^{\prime} \backslash\left\{\left\{u, x_{1}\right\},\left\{v, y_{j}\right\}\right\}$ is a maximal matching of the disjoint union of 2 copies of $P_{3}$ (the path graph with 3 vertices) and $k-2$ copies of the 4-cycle. Hence it follows that $\# \mathcal{M}^{\prime}=2+2+2(k-2)=2 k$. If $\left\{v, y_{j}\right\} \notin \mathcal{M}^{\prime}$ for all $j$, then $\mathcal{M}^{\prime} \backslash\left\{\left\{u, x_{1}\right\}\right\}$ is a maximal matching of the disjoint union of $P_{3}$ and $k-1$ copies of the 4 -cycle. Hence $\# \mathcal{M}^{\prime}=1+1+2(k-1)=2 k$. When $\left\{u, x_{j}\right\},\left\{v, y_{j}\right\} \notin \mathcal{M}$ for all $j, \mathcal{M}^{\prime}$ is a maximal matching of $k$ copies of the 4-cycle. Therefore $\# \mathcal{M}^{\prime}=2 k$.

Finally, we compute $\operatorname{reg}\left(K\left[V_{k}\right] / I\left(H_{k}\right)\right)$. We consider the following decomposition of $H_{k}$ : (a) the 4-cycle with vertices $x_{j}, z_{j 1}, y_{j}, z_{j 2}$ and the edge $\left\{x_{j}, u\right\}$ $(j=1,2, \ldots, k)$; (b) the star graph on $\left\{v, y_{1}, \ldots, y_{k}\right\}$. The edge ideal of each decomposed graph is of regularity 1 . Thus we have $\operatorname{reg}\left(K\left[V_{k}\right] / I\left(H_{k}\right)\right) \leq k+1$ by Lemma 1.8,

For the opposite inequality $\operatorname{reg}\left(K\left[V_{k}\right] / I\left(H_{k}\right)\right) \geq k+1$, we consider the induced subgraph of $H_{k}$ on

$$
W_{k}=\left\{u, v, x_{1}, x_{2}, \ldots, x_{k}, y_{1}, y_{2}, \ldots, y_{k}, z_{11}, z_{21}, \ldots, z_{k 1}\right\} .
$$

We use the labeling of edges of $H_{k}$ as in (1.3). For the sake of simplicity, we use $z_{i}$ (resp. $\left.\mathfrak{g}_{i}, \mathfrak{h}_{i}\right)$ instead of $z_{i 1}$ (resp. $\left.\mathfrak{g}_{i 1}, \mathfrak{h}_{i 1}\right)$ for $i=1,2, \ldots, k$ and denote this graph by $H_{k}^{\prime}$; see Figure 2. By Hochster's formula for Betti numbers (see also [14, Lemma 3.1]), it is enough to prove that $\beta_{2 k+1,3 k+2}\left(K\left[W_{k}\right] / I\left(H_{k}^{\prime}\right)\right) \neq 0$. In order to prove this, we use a Lyubeznik resolution. We identify edges of $H_{k}^{\prime}$ and minimal monomial generators of $I\left(H_{k}^{\prime}\right)$. 


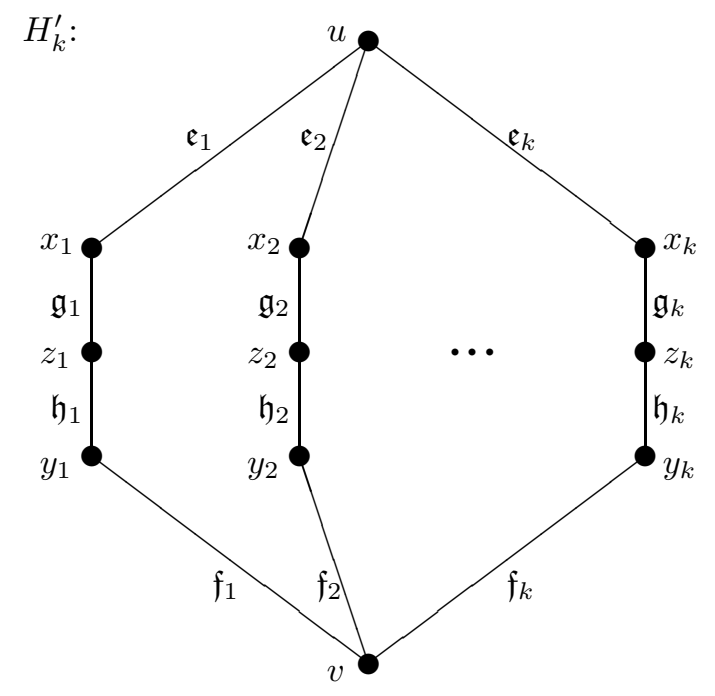

FiguRE 2. The graph $H_{k}^{\prime}$

When $k=2, H_{2}^{\prime}$ is the 8 -cycle. (Hence we know that reg $K\left[W_{2}\right] / I\left(H_{2}^{\prime}\right)=3$.) Let us consider the Lyubeznik resolution of $I\left(H_{2}^{\prime}\right)$ with respect to the following order of edges of $H_{2}^{\prime}$ (which corresponds to the order of minimal monomial generators of $\left.I\left(H_{2}^{\prime}\right)\right)$ :

$$
\mathfrak{e}_{1}, \mathfrak{h}_{1}, \mathfrak{f}_{k}, \mathfrak{g}_{k}, \mathfrak{e}_{k}, \mathfrak{g}_{1}, \mathfrak{f}_{1}, \mathfrak{h}_{k}
$$

We denote the resolution by $\left(\mathcal{L}_{\bullet}^{(2)}, d_{\bullet}^{(2)}\right)$. Then the maximal $L$-admissible symbols are

$$
\left[\mathfrak{e}_{1}, \mathfrak{h}_{1}, \mathfrak{f}_{k}, \mathfrak{g}_{k}, \mathfrak{e}_{k}, \mathfrak{f}_{1}\right],\left[\mathfrak{e}_{1}, \mathfrak{h}_{1}, \mathfrak{f}_{k}, \mathfrak{g}_{k}, \mathfrak{g}_{1}, \mathfrak{h}_{k}\right]
$$

Put

$$
\xi^{(2)}:=\left[\mathfrak{e}_{1}, \mathfrak{h}_{1}, \mathfrak{f}_{k}, \mathfrak{g}_{k}, \mathfrak{e}_{k}\right]-\left[\mathfrak{e}_{1}, \mathfrak{h}_{1}, \mathfrak{f}_{k}, \mathfrak{g}_{k}, \mathfrak{g}_{1}\right] \in \mathcal{L}_{5}^{(2)} .
$$

Then it is easy to see that $1 \otimes \xi^{(2)} \in \operatorname{Ker}\left(1 \otimes d_{5}^{(2)}\right) \backslash \operatorname{Im}\left(1 \otimes d_{6}^{(2)}\right)$. Also $\operatorname{deg} \xi^{(2)}=8$. Therefore we have $\beta_{5,8}\left(K\left[V_{2}\right] / I\left(H_{2}^{\prime}\right)\right) \neq 0$.

Next assume that $k \geq 3$. For each $i=2, \ldots, k-1$, consider the induced subgraph of $H_{k}^{\prime}$ on $\left\{u, x_{i}, z_{i}, y_{i}, v\right\}$. We denote it by $L_{i}^{(k)}$. Also let $C_{8}^{(k)}$ be the induced subgraph of $H_{k}^{\prime}$ on $\left\{u, x_{1}, z_{1}, y_{1}, v, y_{k}, z_{k}, x_{k}\right\}$, which is the 8-cycle. Note that $E\left(H_{k}^{\prime}\right)=\bigcup_{i=2}^{k-1} E\left(L_{i}^{(k)}\right) \cup E\left(C_{8}^{(k)}\right)$.

Let us consider the Lyubeznik resolution of $I\left(L_{i}^{(k)}\right)$ with respect to the following order of edges of $L_{i}^{(k)}: \mathfrak{g}_{i}, \mathfrak{h}_{i}, \mathfrak{f}_{i}, \mathfrak{e}_{i}$. We denote the resolution by $\left(\mathcal{L}_{\bullet}^{(k, i)}, d_{\bullet}^{(k, i)}\right)$. Then the maximal $L$-admissible symbols are $\left[\mathfrak{g}_{i}, \mathfrak{f}_{i}, \mathfrak{e}_{i}\right],\left[\mathfrak{g}_{i}, \mathfrak{h}_{i}, \mathfrak{f}_{i}\right]$ and it is easy to see that $1 \otimes\left[\mathfrak{g}_{i}, \mathfrak{h}_{i}\right] \in \operatorname{Ker}\left(1 \otimes d_{2}^{(k, i)}\right) \backslash \operatorname{Im}\left(1 \otimes d_{3}^{(k, i)}\right)$. Also $\operatorname{deg}\left[\mathfrak{g}_{i}, \mathfrak{h}_{i}\right]=3$.

We also consider the Lyubeznik resolution of $I\left(C_{8}^{(k)}\right)$ with respect to the ordering as in (1.4). Then the same argument with $H_{2}^{\prime}$ is valid. 
Now let us consider the Lyubeznik resolution of $I\left(H_{k}^{\prime}\right)$ with respect to the following order of edges of $H_{k}^{\prime}$ :

$$
\begin{aligned}
\mathfrak{g}_{2}, \mathfrak{h}_{2}, \mathfrak{f}_{2}, \mathfrak{e}_{2}, \\
\ldots, \\
\mathfrak{g}_{k-1}, \mathfrak{h}_{k-1}, \mathfrak{f}_{k-1}, \mathfrak{e}_{k-1}, \\
\quad \mathfrak{e}_{1}, \mathfrak{h}_{1}, \mathfrak{f}_{k}, \mathfrak{g}_{k}, \mathfrak{e}_{k}, \mathfrak{g}_{1}, \mathfrak{f}_{1}, \mathfrak{h}_{k} .
\end{aligned}
$$

We denote the resolution by $\left(\mathcal{L}_{\bullet}^{(k)}, d_{\bullet}^{(k)}\right)$. Note that for $i=1, \ldots, k-2$, the $i$ th row of (1.5) corresponds to $L_{i+1}^{(k)}$ and the last row of (1.5) corresponds to $C_{8}^{(k)}$. These graphs are only connected by the vertices $u, v$. By the definition of the ordering of the minimal monomial generators of $I\left(H_{k}^{\prime}\right)$, it is easy to see that the $L$-admissible symbols of $\left(\mathcal{L}_{\bullet}^{(k)}, d_{\bullet}^{(k)}\right)$ are obtained by each $L$-admissible symbols for $L_{2}^{(k)}, \ldots, L_{k-1}^{(k)}$ and $C_{8}^{(k)}$. A similar claim is true when we consider the maximal $L$-admissible symbols. Put

$$
\begin{aligned}
\xi^{(k)}:= & {\left[\mathfrak{g}_{2}, \mathfrak{h}_{2}, \ldots, \mathfrak{g}_{k-1}, \mathfrak{h}_{k-1}, \mathfrak{e}_{1}, \mathfrak{h}_{1}, \mathfrak{f}_{k}, \mathfrak{g}_{k}, \mathfrak{e}_{k}\right] } \\
& -\left[\mathfrak{g}_{2}, \mathfrak{h}_{2}, \ldots, \mathfrak{g}_{k-1}, \mathfrak{h}_{k-1}, \mathfrak{e}_{1}, \mathfrak{h}_{1}, \mathfrak{f}_{k}, \mathfrak{g}_{k}, \mathfrak{g}_{1}\right]
\end{aligned}
$$

Then $\xi^{(k)} \in\left(\mathcal{L}_{2(k-2)+5}^{(k)}\right)_{3(k-2)+8}$. Also $1 \otimes \xi^{(k)} \in \operatorname{Ker}\left(1 \otimes d_{2(k-2)+5}^{(k)}\right) \backslash \operatorname{Im}(1 \otimes$ $\left.d_{2(k-2)+6}^{(k)}\right)$ follows. Therefore we have $\beta_{2 k+1,3 k+2}\left(K\left[V_{k}\right] / I\left(H_{k}^{\prime}\right)\right) \neq 0$ as desired.

Question 1.11. Can we construct an infinite family of finite simple connected graphs $G$ satisfying ( $v)$ ?

A finite simple connected graph $G$ satisfying the inequalities (v) might be rare. Actually, when the number of vertices of $G$ is at most 7 , there is no such a graph $G$ with (v) except for $C_{5}$.

Proposition 1.12. Let $G$ be a finite simple connected graph with at most 7 vertices. Then $\operatorname{match}(G)=\operatorname{reg} S / I(G)>\operatorname{ind}-\operatorname{match}(G)$ if and only if $G$ is a 5-cycle.

Remark 1.13. After submitting the paper, Biyikoğlu and Civan [3, Theorem 3.17] proved that there is no finite simple connected graph $G$ satisfying (v) except for $C_{5}$.

\section{A graph With A DOMinating INDUCED MATChing}

In [10, the authors studied the Cameron-Walker graphs. In this section, we treat some classes of graphs which contain Cameron-Walker graphs as a subclass and investigate these combinatorial properties.

We first recall some definitions on graphs.

Let $G$ be a finite simple graph on the vertex set $V$. Let $W$ be a subset of $V$. We denote by $G_{W}$ the induced subgraph of $G$ on $W$ : the vertex set of $G_{W}$ is $W$ and the edge set of $G_{W}$ consists of all edges of $G$ which are contained in $W$. We write $G \backslash W$ instead of $G_{V \backslash W}$. In particular, when $W=\{x\}$, consisting of 1 vertex, we write $G \backslash x$ instead of $G \backslash\{x\}$. For a vertex $x \in V$, we denote by $N_{G}(x)$ the set of neighbours of $x$. Also we set $N_{G}[x]:=N_{G}(x) \cup\{x\}$. The degree of $x$ is defined by $\operatorname{deg}_{G}(x):=\# N_{G}(x)$. For a subset $W \subset V$, we set $N_{G}(W)=\bigcup_{x \in W} N_{G}(x)$ and $N_{G}[W]=\bigcup_{x \in W} N_{G}[x]$. We sometimes omit the lower subscript $G$ on these notation if there is no fear of confusion. 
A subset $W \subset V$ is called independent if no two vertices of $W$ are adjacent in $G$. An independent set $W$ is said to be maximal if there is no independent set of $G$ which properly contains $W$. Also a subset $C \subset V$ is called a vertex cover of $G$ if all edges of $G$ meet with $C$. A vertex cover $C$ is said to be minimal if there is no vertex cover of $G$ which is properly contained in $C$. Note that $C$ is a minimal vertex cover of $G$ if and only if $V \backslash C$ is a maximal independent set of $G$. A graph is said to be unmixed if all minimal vertex covers (equivalently, all maximal independent sets) of $G$ have the same cardinality. When $G$ is unmixed, the edge ideal $I(G)$ is height unmixed.

An edge of $G$ is called a leaf edge if it contains a degree 1 vertex. Also a triangle of $G$ is called a pendant triangle if its two vertices are of degree 2 and the rest vertex is of degree more than 2.

A Cameron-Walker graph $G$ satisfies the equalities (i) in the previous section:

$$
\operatorname{ind}-\operatorname{match}(G)=\operatorname{reg} S / I(G)=\min -\operatorname{match}(G)=\operatorname{match}(G)
$$

Recall that a Cameron-Walker graph $G$ consists of a connected bipartite graph with the vertex partition $X \sqcup Y$ such that there is at least one leaf edge attached to each vertex $x_{i} \in X$ and that there may be possibly some pendant triangles attached to a vertex $y_{j} \in Y$. Choose one leaf edge which contains $x_{i}$ for each $x_{i} \in X$. Then these edges and the edges consisting of two degree 2 vertices of all pendant triangles form an induced matching of $G$. It also forms a maximal matching of $G$. Thus for a Cameron-Walker graph $G$, there exists an induced matching of $G$ which is also a maximal matching of $G$. Such a matching is called a dominating induced matching or an efficient edge domination set. There exists a graph which does not have a dominating induced matching. For example, let $G_{0}$ be the graph on the vertex set $\{1,2, \ldots, 6\}$ with edges $\{1,2\},\{2,3\},\{3,4\},\{4,5\},\{3,6\}$. Then it is easy to see that an induced matching consisting of one edge is not a maximal matching of $G_{0}$. Other induced matching of $G_{0}$ is only $\{\{1,2\},\{4,5\}\}$, which is also not a maximal matching; $\{\{1,2\},\{4,5\},\{3,6\}\}$ is a matching of $G_{0}$. On graph theory, it has been studied the problem of determining whether a given finite simple graph has a dominating induced matching. This problem is known to be NP-complete in general; see e.g., 5, 16.

A graph with a dominating induced matching is characterized as follows. It is easy to check but we give a proof of this for the completeness.

Proposition 2.1 (cf. [16, p.2]). Let $G$ be a finite simple graph on $V$. Then $G$ has a dominating induced matching if and only if there is an independent set $W$ such that $G \backslash W$ is a disjoint union of edges. When this is the case, the set of edges of $G \backslash W$ forms a dominating induced matching of $G$.

Proof. Let $\mathcal{M}=\left\{e_{1}, e_{2}, \ldots, e_{s}\right\}$ be a dominating induced matching of $G$. Let $W$ be the set of vertices which do not appear in each $e_{i}$. Then $W$ is an independent set of $G$ since $\mathcal{M}$ is a maximal matching.

For example, the path graph $P_{6 n}$ with $6 n$ vertices and the $6 n$-cycle $C_{6 n}$ have dominating induced matchings; for instance, take $W=\{3,6, \ldots, 6 n\}$.

Let $G$ be a finite simple graph. Since ind-match $(G) \leq \min -\operatorname{match}(G)$ holds in general, if $G$ has a dominating induced matching, then ind-match $(G)=\min -\operatorname{match}(G)$ holds. 
Remark 2.2. For a finite simple graph $G$, the inequality ind-match $(G) \leq \min -\operatorname{match}(G)$ follows via reg $S / I(G)$; see (1.1).

We prove this inequality by pure combinatorics. Let $\mathcal{M}=\left\{e_{1}, \ldots, e_{s}\right\}$ be an induced matching of $G$ and $\mathcal{M}^{\prime}=\left\{e_{1}^{\prime}, \ldots, e_{t}^{\prime}\right\}$ a maximal matching of $G$. Then for each $e_{k} \in \mathcal{M}$, there exists $e_{i_{k}}^{\prime} \in \mathcal{M}^{\prime}$ with $e_{i_{k}}^{\prime} \cap e_{k} \neq \emptyset$ because of the maximality of $\mathcal{M}^{\prime}$. Since $\mathcal{M}$ is an induced matching, it follows that $i_{k} \neq i_{j}$ if $k \neq j$. Therefore we have ind-match $(G) \leq \min -\operatorname{match}(G)$.

We next characterize a finite simple graph $G$ with ind-match $(G)=\min -\operatorname{match}(G)$.

Theorem 2.3. Let $G$ be a finite simple graph on $V$. Then $G$ satisfies ind-match $(G)=$ $\min -\operatorname{match}(G)$ if and only if the vertex set $V$ can be partitioned as

$$
V=\left\{v_{i 1}, v_{i 2}: i=1,2, \ldots, \alpha+\beta\right\} \sqcup\left\{z_{1}, \ldots, z_{\alpha}\right\} \sqcup\left\{w_{1}, \ldots, w_{\gamma}\right\},
$$

where $\alpha, \beta, \gamma$ are non-negative integers, so that the edge set of $G$ is of the following form

$$
\left\{e_{i}: i=1,2, \ldots, \alpha+\beta\right\} \cup\left\{e_{i}^{\prime}: i=1,2, \ldots, \alpha\right\} \cup E^{\prime},
$$

where we set $e_{i}=\left\{v_{i 1}, v_{i 2}\right\}(i=1,2, \ldots, \alpha+\beta)$ and $e_{i}^{\prime}=\left\{v_{i 1}, z_{i}\right\}(i=1,2, \ldots, \alpha)$, and an edge in $E^{\prime}$ is one of the following:

(i) an edge containing $z_{i}(i=1,2, \ldots, \alpha)$;

(ii) an edge consisting of an end vertex of $e_{i}(i=\alpha+1, \alpha+2, \ldots, \alpha+\beta)$ and $w_{j}(j=1,2, \ldots, \gamma)$;

(iii) an edge consisting of $v_{i 1}(i=1,2, \ldots, \alpha)$ and $w_{j}(j=1,2, \ldots, \gamma)$.

Proof. ("If") It is easy to see that $e_{1}, \ldots, e_{\alpha+\beta}$ form an induced matching of $G$. Hence we have $\alpha+\beta \leq$ ind-match $(G)$. On the other hand, $e_{1}^{\prime}, \ldots, e_{\alpha}^{\prime}, e_{\alpha+1}, \ldots, e_{\alpha+\beta}$ form a maximal matching of $G$ since the rest vertices are $v_{12}, \ldots, v_{\alpha 2}, w_{1}, \ldots, w_{\gamma}$. Hence we have min-match $(G) \leq \alpha+\beta$. By combining these inequalities, we have ind-match $(G)=\min -\operatorname{match}(G)=\alpha+\beta$.

("Only If") Put $s=$ ind-match $(G)=\min -\operatorname{match}(G)$. Let $\mathcal{M}=\left\{e_{1}, \ldots, e_{s}\right\}$ be an induced matching of $G$ with cardinality $s$ and $\mathcal{M}^{\prime}=\left\{e_{1}^{\prime}, \ldots, e_{s}^{\prime}\right\}$ a maximal matching of $G$ with cardinality $s$. As noted in Remark 2.2 for each $e_{k} \in \mathcal{M}$, there exists an edge $e_{i_{k}}^{\prime} \in \mathcal{M}^{\prime}$ with $e_{i_{k}}^{\prime} \cap e_{k} \neq \emptyset$, and $i_{k} \neq i_{j}$ if $k \neq j$. Also since $\# \mathcal{M}=s=\operatorname{ind}-\operatorname{match}(G), i_{k}$ is uniquely determined by $k$. Therefore we may assume that $e_{k}^{\prime} \neq e_{k}$ for $k=1, \ldots, \alpha(0 \leq \alpha \leq s)$ and $e_{k}^{\prime}=e_{k}$ for $k=\alpha+1, \ldots, s$. Set $z_{k}=e_{k}^{\prime} \backslash e_{k}(k=1, \ldots, \alpha)$ and $W=V \backslash\left(\bigcup_{k=1}^{s} e_{k} \cup\left\{z_{1}, \ldots, z_{\alpha}\right\}\right)$. Then it is easy to see that

$$
V=\left(\bigcup_{k=1}^{s} e_{k}\right) \sqcup\left\{z_{1}, \ldots, z_{\alpha}\right\} \sqcup W
$$

is a desired partition.

As mentioned before Remark 2.2, if $G$ has a dominating induced matching, then ind-match $(G)=\min -\operatorname{match}(G)$ holds. But the converse is false; the graph $G_{0}$ (see the beginning of this section) does not have a dominating induced matching, but ind-match $\left(G_{0}\right)=\min$-match $\left(G_{0}\right)=2$; for example, $\{\{2,3\},\{4,5\}\}$ is a maximal matching with cardinality 2 .

Now we return to the graph with a dominating induced matching. We consider the problem which graph with a dominating induced matching is unmixed. Let $G$ be a finite simple graph on the vertex set $V$ with a dominating induced matching. 
Then $V$ can be decomposed as $W \sqcup M$ where $W$ is an independent set of $G$ and $G_{M}$ consists of $m:=\min -\operatorname{match}(G)$ disconnected edges $\left\{x_{j 1}, x_{j 2}\right\}, j=1, \ldots, m$. Set

$$
W_{0}:=\{w \in W: w \text { is not an isolated vertex of } G\}
$$

Also set

$$
\begin{aligned}
& m_{1}:=\#\left\{j: \operatorname{deg}_{G} x_{j 1}=1 \text { or } \operatorname{deg}_{G} x_{j 2}=1\right\} \\
& m_{2}:=\#\left\{j: \operatorname{deg}_{G} x_{j 1} \geq 2 \text { and } \operatorname{deg}_{G} x_{j 2} \geq 2\right\} .
\end{aligned}
$$

Note that $m_{1}+m_{2}=m$. For a subset $U \subset V$, we denote by $I N_{G}(U, W)$ (or $I N(U, W)$ if there is no fear of confusion), the set of isolated vertices of $G \backslash N_{G}[U]$ which are contained in $W_{0}$.

Let $M_{2}$ be a subset of $M$ satisfying the following 2 conditions:

$(* 1) \#\left(M_{2} \cap\left\{x_{j 1}, x_{j 2}\right\}\right) \leq 1$ for all $j=1, \ldots, m$;

$(* 2) \operatorname{deg}_{G} x \geq 2$ for all $x \in M_{2}$,

Then $G^{\prime}:=G \backslash N_{G}\left[M_{2}\right]$ has a dominating induced matching if $G^{\prime}$ is not an edgeless graph. Indeed $V^{\prime}:=V\left(G^{\prime}\right)=V \backslash N_{G}\left[M_{2}\right]$, the vertex set of $G^{\prime}$, can be decomposed as $W^{\prime} \sqcup M^{\prime}$, where $W^{\prime}=W \backslash N_{G}\left[M_{2}\right]$ and $M^{\prime}=M \backslash N_{G}\left[M_{2}\right]$. We use notation $m_{1}^{\prime}, m_{2}^{\prime}, W_{0}^{\prime}$ for $G^{\prime}$ with respect to this decomposition of $V^{\prime}$ with a similar meaning to $G$. If $G^{\prime}$ is an edgeless graph, then we set $m_{1}^{\prime}=m_{2}^{\prime}=0$ and $W_{0}^{\prime}=\emptyset$.

Theorem 2.4. We use the same notation as above. Let $G$ be a finite simple graph on $V$ with a dominating induced matching. Then $G$ is unmixed if and only if for some (and then all) decomposition $V=W \sqcup M$, the following condition (b) is satisfied:

Condition (b): For each subset $M_{2} \subset M$ with the properties $(* 1),(* 2)$, the following 2 conditions are satisfied:

$$
\begin{aligned}
& \text { (b1) } m_{2}-m_{2}^{\prime}=\# N_{G}\left(M_{2}\right)-\# M_{2} . \\
& \text { (b2) } \# W_{0} \leq 2 m_{2}-\# N_{G}\left(M_{2}\right)+\# M_{2}+\# I N_{G}\left(M_{2}, W\right) .
\end{aligned}
$$

Remark 2.5. We use the same notation as in Theorem 2.4.

(1) The empty set $M_{2}=\emptyset$ is regarded as satisfying $(* 1)$ and $(* 2)$. Then (b1) is satisfied as both-hand sides are 0 . Also (b2) must be $\# W_{0} \leq 2 m_{2}$. Indeed if $G$ is unmixed, this inequality holds; see Lemma 2.6 below.

(2) The left-hand side of (b1) is equal to the cardinality of the following set:

$\mathcal{I}_{G, M_{2}}:=\left\{\begin{array}{ll} & \operatorname{deg}_{G} x_{j 1} \geq 2, \operatorname{deg}_{G} x_{j 2} \geq 2, \text { and one of the following is satisfied: } \\ & \left(\text { i) } M_{2} \cap\left\{x_{j 1}, x_{j 2}\right\} \neq \emptyset ;\right. \\ & \text { (ii) } N_{G}\left(x_{j 1}\right) \backslash\left\{x_{j 2}\right\} \subset N_{G}\left(M_{2}\right) ; \\ & (\text { iii }) N_{G}\left(x_{j 2}\right) \backslash\left\{x_{j 1}\right\} \subset N_{G}\left(M_{2}\right)\end{array}\right\}$.

Indeed, $m_{2}-m_{2}^{\prime}$ counts the number of edges $\left\{x_{j 1}, x_{j 2}\right\}$ with $\operatorname{deg}_{G} x_{j 1} \geq 2$ and $\operatorname{deg}_{G} x_{j 2} \geq 2$ such that both $x_{j 1}$ and $x_{j 2}$ are not vertices of $G^{\prime}$ or one of $\operatorname{deg}_{G^{\prime}} x_{j 1}=1$ and $\operatorname{deg}_{G^{\prime}} x_{j 2}=1$ holds.

We first prove the following lemma.

Lemma 2.6. We use the same notation as above. Let $G$ be a finite simple graph on $V$ with a dominating induced matching: $V=W \sqcup M$. If $G$ is unmixed, then $\# W_{0} \leq 2 m_{2}$. 
Proof. Let us consider the following subset of $V$ :

$$
C_{0}:=\left(\bigcup_{\substack{j \\ \operatorname{deg}_{G} x_{j 1} \geq 2, \operatorname{deg}_{G} x_{j 2} \geq 2}}\left\{x_{j 1}, x_{j 2}\right\}\right) \cup\left(\bigcup_{\substack{j \\ \operatorname{leg}_{G} x_{j k} \geq \operatorname{deg}_{G} x_{j \ell}=1}}\left\{x_{j k}\right\}\right) .
$$

Then $C_{0}$ is a minimal vertex cover of $G$. Since $G$ is unmixed, we have height $I(G)=$ $m_{1}+2 m_{2}$. By Gitler and Valencia [8, Corollary 3.4], we have 2 height $I(G) \geq$ $\# W_{0}+\# M$. Note that $\# M=2\left(m_{1}+m_{2}\right)$. Hence

$$
\# W_{0} \leq 2 \text { height } I(G)-\# M=2\left(m_{1}+2 m_{2}\right)-2\left(m_{1}+m_{2}\right)=2 m_{2} .
$$

Now we prove Theorem 2.4

Proof of Theorem 2.4. We first assume that $G$ is unmixed. Let $W \sqcup M$ be a decomposition of $V$ where $W$ is an independent set of $G$ and $G_{M}$ consists of disconnected edges $\left\{x_{j 1}, x_{j 2}\right\}, j=1, \ldots, m$. Since $C_{0}$ in (2.2) is a minimal vertex cover of $G$, the cardinality of any minimal vertex cover of $G$ is $m_{1}+2 m_{2}$. Let $M_{2}$ be a subset of $M$ satisfying the conditions $(* 1)$ and $(* 2)$. Then $G^{\prime}=G \backslash N_{G}\left[M_{2}\right]$ is an edgeless graph or a graph with dominating induced matching as noted before Theorem 2.4. Considering the minimal vertex cover of $G$ which is disjoint with $M_{2}$, we have $m_{1}+2 m_{2}=\# N_{G}\left(M_{2}\right)+m_{1}^{\prime}+2 m_{2}^{\prime}$. (If $G^{\prime}$ is an edgeless graph, then we consider both $m_{1}^{\prime}$ and $m_{2}^{\prime}$ as 0 .) Also focusing on the number of edges of $G_{M}$, we have $m_{1}+m_{2}=\# M_{2}+m_{1}^{\prime}+m_{2}^{\prime}$. Then we have

$$
m_{2}-m_{2}^{\prime}=\# N_{G}\left(M_{2}\right)-\# M_{2} .
$$

Hence (b1) holds. Also note that when $G$ is unmixed, $G^{\prime}$ is also unmixed since the union of a minimal vertex cover of $G^{\prime}$ and $N_{G}\left(M_{2}\right)$ is a minimal vertex cover of $G$. Then by Lemma 2.6, we have $\# W_{0}^{\prime} \leq 2 m_{2}^{\prime}$. Since

$$
\# W_{0}^{\prime}=\# W_{0}-\left(\# N_{G}\left(M_{2}\right)-\# M_{2}\right)-\# I N_{G}\left(M_{2}, W\right)
$$

and (2.3), we have

$$
\begin{aligned}
\# W_{0} & =\# W_{0}^{\prime}+\left(\# N_{G}\left(M_{2}\right)-\# M_{2}\right)+\# I N_{G}\left(M_{2}, W\right) \\
& \leq 2 m_{2}^{\prime}+\left(\# N_{G}\left(M_{2}\right)-\# M_{2}\right)+\# I N_{G}\left(M_{2}, W\right) \\
& =2\left(m_{2}-\# N_{G}\left(M_{2}\right)+\# M_{2}\right)+\left(\# N_{G}\left(M_{2}\right)-\# M_{2}\right)+\# I N_{G}\left(M_{2}, W\right) \\
& =2 m_{2}-\# N_{G}\left(M_{2}\right)+\# M_{2}+\# I N_{G}\left(M_{2}, W\right) .
\end{aligned}
$$

Thus (b2) also holds.

We next assume that the decomposition $V=W \sqcup M$ satisfies the condition (b). As noted in Remark 2.5, the inequality $\# W_{0} \leq 2 m_{2}$ is satisfied. We use induction on $m$.

When $m=1$, there are 2 cases: $\left(m_{1}, m_{2}\right)=(1,0),(0,1)$.

If $\left(m_{1}, m_{2}\right)=(1,0)$, then $\# W_{0} \leq 2 m_{2}=0$. Therefore it follows that $G$ is a graph consisting of a single edge with isolated vertices and thus $G$ is unmixed.

If $\left(m_{1}, m_{2}\right)=(0,1)$, then $\# W_{0} \leq 2 m_{2}=2$. Also, since $m_{2}=1>0$, we have $\# W_{0}>0$. Hence $\# W_{0}=1,2$. We first assume that $\# W_{0}=1$. Since $\operatorname{deg}_{G} x_{11}, \operatorname{deg}_{G} x_{12} \geq 2$, it follows that $G$ is a triangle with isolated vertices. Thus 
it is unmixed. We next assume that $\# W_{0}=2$. Put $W_{0}=\left\{w_{1}, w_{2}\right\}$. Take $M_{2}=$ $\left\{x_{12}\right\}$. Then $M_{2}$ satisfies the conditions $(* 1)$ and $(* 2)$. By (b2), we have

$$
\begin{aligned}
2=\# W_{0} & \leq 2 m_{2}-\# N_{G}\left(M_{2}\right)+\# M_{2}+\# I N_{G}\left(M_{2}, W\right) \\
& =2-\# N_{G}\left(x_{12}\right)+1+\# I N_{G}\left(\left\{x_{12}\right\}, W\right) .
\end{aligned}
$$

Hence $\operatorname{deg}_{G} x_{12}=\# N_{G}\left(x_{12}\right) \leq \# I N_{G}\left(\left\{x_{12}\right\}, W\right)+1$. Note that $\operatorname{deg}_{G} x_{12}=2,3$. If $\operatorname{deg}_{G} x_{12}=3$, then $\left\{x_{12}, w_{1}\right\},\left\{x_{12}, w_{2}\right\} \in E(G)$ and $I N_{G}\left(\left\{x_{12}\right\}, W\right)=\emptyset$. This contradicts to $\operatorname{deg}_{G} x_{12} \leq \# I N_{G}\left(\left\{x_{12}\right\}, W\right)+1$. Hence $\operatorname{deg}_{G} x_{12}=2$. The same is true for $x_{11}$. Therefore we conclude that the edge set of $G_{W_{0} \cup M}$ is, by renumbering the vertices, $\left\{\left\{x_{11}, w_{1}\right\},\left\{x_{12}, w_{2}\right\},\left\{x_{11}, x_{12}\right\}\right\}$, and thus $G$ is unmixed.

We next assume that $m \geq 2$. Since $C_{0}$ is a minimal vertex cover of $G$ with cardinality $m_{1}+2 m_{2}$, it is sufficient to prove that the cardinality of any minimal vertex cover of $G$ is $m_{1}+2 m_{2}$. Let $C$ be a minimal vertex cover of $G$ which is not of the form $C_{0}$. Then there exists a vertex in $M$, say $x_{m 2}$, with $x_{m 2} \notin C$ such that $\operatorname{deg}_{G} x_{m 2} \geq 2$. Then $N_{G}\left(x_{m 2}\right) \subset C$. As noted in Lemma 2.7 below, we have that $C \backslash N_{G}\left(x_{m 2}\right)$ is a minimal vertex cover of $G \backslash N_{G}\left[x_{m 2}\right]$.

Put $G^{\prime \prime}:=G \backslash N_{G}\left[x_{m 2}\right]$. Then $G^{\prime \prime}$ is also a graph with a dominating induced matching. Let $W^{\prime \prime} \sqcup M^{\prime \prime}$ be the decomposition of the vertex set $V^{\prime \prime}=V\left(G^{\prime \prime}\right)$ induced by the decomposition $V=W \sqcup M$. Note that $m_{1}^{\prime \prime}+m_{2}^{\prime \prime}=m^{\prime \prime}=m-1$. Then it is sufficient to prove that $V^{\prime \prime}=W^{\prime \prime} \sqcup M^{\prime \prime}$ also satisfies the condition (b) for $G^{\prime \prime}$. Indeed, when this is the case, it follows that $G^{\prime \prime}$ is unmixed by inductive hypothesis. Therefore

$$
\#\left(C \backslash N_{G}\left(x_{m 2}\right)\right)=m_{1}^{\prime \prime}+2 m_{2}^{\prime \prime} .
$$

Consider the condition (b) with $\left\{x_{m 2}\right\}$. By (b1), we have

$$
m_{2}-m_{2}^{\prime \prime}=\# N_{G}\left(x_{m 2}\right)-1 \text {. }
$$

Hence

$$
\begin{aligned}
\# C & =\#\left(C \backslash N_{G}\left(x_{m 2}\right)\right)+\# N_{G}\left(x_{m 2}\right) \\
& =\left(m_{1}^{\prime \prime}+2 m_{2}^{\prime \prime}\right)+\left(m_{2}-m_{2}^{\prime \prime}+1\right) \\
& =m_{1}^{\prime \prime}+m_{2}^{\prime \prime}+1+m_{2} \\
& =m+m_{2} \\
& =m_{1}+2 m_{2},
\end{aligned}
$$

as required.

Now we prove that $V^{\prime \prime}=W^{\prime \prime} \sqcup M^{\prime \prime}$ also satisfies the condition (b) for $G^{\prime \prime}$.

Let $M_{2}^{\prime \prime}$ be a subset of $M^{\prime \prime}$ satisfying $(* 1)$ and $(* 2)$ for $G^{\prime \prime}$. We need to prove that (b1) and (b2) are satisfied. In order to prove (b1), we use the description of the left-hand side of (b1) as in Remark 2.5 (2). Put $M_{2}=M_{2}^{\prime \prime} \cup\left\{x_{m 2}\right\}$. Note that $M_{2}$ and $\left\{x_{m 2}\right\}$ satisfy $(* 1)$ and $(* 2)$ for $G$. Also note that the right-hand side of (b1) for $\left(G, M_{2}\right)$ is

$$
\# N_{G}\left(M_{2}\right)-\# M_{2}=\left(\# N_{G^{\prime \prime}}\left(M_{2}^{\prime \prime}\right)-\# M_{2}^{\prime \prime}\right)+\left(\# N_{G}\left(x_{m 2}\right)-1\right)
$$

because $\# M_{2}=\# M_{2}^{\prime \prime}+1$ and $\# N_{G}\left(M_{2}\right)=\# N_{G^{\prime \prime}}\left(M_{2}^{\prime \prime}\right)+\# N_{G}\left(x_{m 2}\right)$.

Now, let $j$ be an index with $\operatorname{deg}_{G} x_{j 1} \geq 2$ and $\operatorname{deg}_{G} x_{j 2} \geq 2$. Recall that the left-hand side of (b1) for $\left(G, M_{2}\right)$ is the number of $j$ for which one of the condition (i), (ii), (iii) inside $\mathcal{I}_{G, M_{2}}$ is satisfied. We compare the satisfaction of the condition for the pair $\left(G, M_{2}\right)$ with that for the pair $\left(G^{\prime \prime}, M_{2}^{\prime \prime}\right)$. If $j \neq m$, and $\operatorname{deg}_{G^{\prime \prime}} x_{j 1} \geq 2$ and $\operatorname{deg}_{G^{\prime \prime}} x_{j 2} \geq 2$, then the satisfaction of each of the conditions (i), (ii), (iii) inside 
$\mathcal{I}_{G, M_{2}}$ and $\mathcal{I}_{G^{\prime \prime}, M_{2}^{\prime \prime}}$ is equivalent. If $j=m$, then note that $x_{m 2} \in\left\{x_{m 2}\right\} \subset M_{2}$, that is $\left\{x_{m 2}\right\} \cap\left\{x_{m 1}, x_{m 2}\right\} \neq \emptyset$ as well as $M_{2} \cap\left\{x_{m 1}, x_{m 2}\right\} \neq \emptyset$, which corresponds to the condition (i) inside $\mathcal{I}_{G,\left\{x_{m 2}\right\}}, \mathcal{I}_{G, M_{2}}$, respectively. If $j \neq m$, and $\operatorname{deg}_{G^{\prime \prime}} x_{j 1} \leq 1$ or $\operatorname{deg}_{G^{\prime \prime}} x_{j 2} \leq 1$, then one of the following is satisfied:

$$
\begin{aligned}
& N_{G}\left(x_{j 1}\right) \backslash\left\{x_{j 2}\right\} \subset N_{G}\left(x_{m 2}\right) \subset N_{G}\left(M_{2}\right), \\
& N_{G}\left(x_{j 2}\right) \backslash\left\{x_{j 1}\right\} \subset N_{G}\left(x_{m 2}\right) \subset N_{G}\left(M_{2}\right) .
\end{aligned}
$$

These correspond to the conditions (ii), (iii) inside $\mathcal{I}_{G,\left\{x_{m 2}\right\}}, \mathcal{I}_{G, M_{2}}$. Note that when $j \neq m$, and $\operatorname{deg}_{G^{\prime \prime}} x_{j 1} \geq 2$ and $\operatorname{deg}_{G^{\prime \prime}} x_{j 2} \geq 2$, the cases (i), (ii), (iii) inside $\mathcal{I}_{G,\left\{x_{m 2}\right\}}$ do not occur. Combining these with Remark 2.5 (2), we have that the lefthand-side of (b1) for $M_{2}$ with respect to $G$ is equal to the sum of the lefthandside of (b1) for $\left\{x_{m 2}\right\}$ with respect to $G$ and the lefthand-side of (b1) for $M_{2}^{\prime \prime}$ with respect to $G^{\prime \prime}$. Hence by assumption for $G$, we have that the lefthand-side of (b1) for $M_{2}^{\prime \prime}$ with respect to $G^{\prime \prime}$ is equal to

$$
\left(\# N_{G}\left(M_{2}\right)-\# M_{2}\right)-\left(\# N_{G}\left(\left\{x_{m 2}\right\}\right)-\#\left\{x_{m 2}\right\}\right)
$$

By (2.4), it is equal to

$$
\# N_{G^{\prime \prime}}\left(M_{2}^{\prime \prime}\right)-\# M_{2}^{\prime \prime}
$$

as desired.

Finally we prove the inequality (b2) for $M_{2}^{\prime \prime}$ with respect to $G^{\prime \prime}$. Let $W_{0}^{\prime \prime}$ be the set of vertices in $V^{\prime \prime} \cap W^{\prime \prime}=V^{\prime \prime} \cap W$ which are not isolated in $G^{\prime \prime}$. Then

$$
\# W_{0}^{\prime \prime}=\# W_{0}-\left(\# N_{G}\left(x_{m 2}\right)-1\right)-\# I N_{G}\left(\left\{x_{m 2}\right\}, W\right) .
$$

Also

$$
\begin{aligned}
\# N_{G^{\prime \prime}}\left(M_{2}^{\prime \prime}\right) & =\# N_{G}\left(M_{2}\right)-\# N_{G}\left(x_{m 2}\right), \\
\# I N_{G^{\prime \prime}}\left(M_{2}^{\prime \prime}, W^{\prime \prime}\right) & =\# I N_{G}\left(M_{2}, W\right)-\# I N_{G}\left(\left\{x_{m 2}\right\}, W\right) .
\end{aligned}
$$

Furthermore, it follows from the assumption (b1) for $\left\{x_{m 2}\right\}$ with respect to $G$ that $m_{2}^{\prime \prime}=m_{2}-\# N_{G}\left(x_{m 2}\right)+1$. Then

$$
\begin{aligned}
& 2 m_{2}^{\prime \prime}-\# N_{G^{\prime \prime}}\left(M_{2}^{\prime \prime}\right)+\# M_{2}^{\prime \prime}+\# I N_{G^{\prime \prime}}\left(M_{2}^{\prime \prime}, W^{\prime \prime}\right)-\# W_{0}^{\prime \prime} \\
& =2\left(m_{2}-\# N_{G}\left(x_{m 2}\right)+1\right)-\left(\# N_{G}\left(M_{2}\right)-\# N_{G}\left(x_{m 2}\right)\right)+\left(\# M_{2}-1\right) \\
& +\left(\# I N_{G}\left(M_{2}, W\right)-\# I N_{G}\left(\left\{x_{m 2}\right\}, W\right)\right)-\left(\# W_{0}-\left(\# N_{G}\left(x_{m 2}\right)-1\right)-\# I N_{G}\left(\left\{x_{m 2}\right\}, W\right)\right) \\
& =2 m_{2}-\# N_{G}\left(M_{2}\right)+\# M_{2}+\# I N_{G}\left(M_{2}, W\right)-\# W_{0} \geq 0
\end{aligned}
$$

by the assumption (b2) for $M_{2}$ with respect to $G$. Hence (b2) for $M_{2}^{\prime \prime}$ with respect to $G^{\prime \prime}$ is also satisfied as desired.

Lemma 2.7. $C \backslash N_{G}\left(x_{m 2}\right)$ is a minimal vertex cover of $G \backslash N_{G}\left[x_{m 2}\right]$.

Proof. We first prove that $C \backslash N_{G}\left(x_{m 2}\right)$ is a vertex cover of $G \backslash N_{G}\left[x_{m 2}\right]$. Let $e$ be an edge of $G \backslash N_{G}\left[x_{m 2}\right]$. Then $e \cap N_{G}\left[x_{m 2}\right]=\emptyset$. Also, since $C$ is a vertex cover of $G$, it follows that $e \cap C \neq \emptyset$. Combining these facts we have $e \cap\left(C \backslash N_{G}\left(x_{m 2}\right)\right) \neq \emptyset$.

We next prove the minimality of $C \backslash N_{G}\left(x_{m 2}\right)$. Assume that $C^{\prime} \subsetneq C \backslash N_{G}\left(x_{m 2}\right)$ is a vertex cover of $G \backslash N_{G}\left[x_{m 2}\right]$. Then $C^{\prime} \cup N_{G}\left(x_{m 2}\right) \subsetneq C$. We derive a contradiction by proving that $C^{\prime \prime}:=C^{\prime} \cup N_{G}\left(x_{m 2}\right)$ is a vertex cover of $G$.

Let $e$ be an edge of $G$. If $e \cap N_{G}\left(x_{m 2}\right) \neq \emptyset$, then $e \cap C^{\prime \prime} \neq \emptyset$. If $e \cap N_{G}\left(x_{m 2}\right)=\emptyset$, then $x_{m 2} \notin e$ and $e$ is an edge of $G \backslash N_{G}\left[x_{m 2}\right]$. Since $C^{\prime}$ is a vertex cover of $G \backslash N_{G}\left[x_{m 2}\right]$, it follows that $e \cap C^{\prime} \neq \emptyset$. Therefore $e \cap C^{\prime \prime} \neq \emptyset$. 
A graph $G$ is called forest if $G$ has no cycle. The chordalness of a graph with a dominating induced matching is characterized as follows:

Theorem 2.8. Let $G$ be a finite simple graph on $V$ with a dominating induced matching. Let

$$
\mathcal{M}=\left\{\left\{x_{j 1}, x_{j 2}\right\}: i=1,2, \ldots, m\right\}
$$

be a matching of $G$ so that

$$
W=V \backslash \bigcup_{j=1}^{m}\left\{x_{j 1}, x_{j 2}\right\}
$$

is an independent set of $G$.

Let $\widetilde{G}$ be the graph obtained by identifying $x_{j 1}$ and $x_{j 2}$ for $j=1,2, \ldots, m$. That is $\widetilde{G}$ is a graph on the vertex set $\widetilde{V}:=W \cup\left\{x_{1}, \ldots, x_{m}\right\}$ with the edge set

$$
E(\widetilde{G})=\left\{\left\{w, x_{j}\right\}:\left\{w, x_{j 1}\right\} \in E(G) \text { or }\left\{w, x_{j 2}\right\} \in E(G)\right\} .
$$

Then $G$ is chordal if and only if $\widetilde{G}$ is a forest.

Proof. We first prove that if $G$ is not chordal, then $\widetilde{G}$ is not a forest, in other words, $\widetilde{G}$ has a cycle. Assume that $G$ has a chordless cycle $C$ of length $\ell$ with $\ell>3$. Let $\widetilde{C}$ be the subgraph of $\widetilde{G}$ obtained from $C$ by the same operation as we obtain $\widetilde{G}$ from $G$. If there is no $j$ such that both of $x_{j 1}, x_{j 2}$ are vertices of $C$, then $\widetilde{C}$ is also a cycle. Hence $\widetilde{G}$ has a cycle. If both of $x_{j 1}, x_{j 2}$ are vertices of $C$, then these must be adjacent in $C$ because $C$ is a chordless cycle. Since $\ell>3$, the other adjacent vertices $y_{i_{1}}, y_{i_{2}}$ of $x_{j 1}, x_{j 2}$ are different. Note that $y_{i_{1}}, y_{i_{2}} \in W$. Then $y_{i_{1}}, y_{i_{2}}, x_{j}$ are vertices of $\widetilde{C}$. It then follows that $\widetilde{C}$ is a cycle of $\widetilde{G}$.

Next suppose that $G$ is chordal. Assume that $\widetilde{G}$ has a cycle. Let $\widetilde{C}$ be a minimal cycle of $\widetilde{G}$ and let $\ell$ be the length of $\widetilde{C}$. Since $\widetilde{G}$ is a bipartite graph, $\ell$ must be even and thus $\ell \geq 4$. Let $C$ be a cycle of $G$ corresponding to $\widetilde{C}$ with the minimum length. Then the length of $C$ is greater than or equal to $\ell \geq 4$. Since $G$ is chordal, $C$ must have a chord $e$. We may assume that $e=\left\{w, x_{j 1}\right\}$ where $w \in W$. Since $C$ is a cycle, there are two paths from $w$ to $x_{j 1}$; we take with the shorter length; let $y_{0}=w, y_{1}, y_{2}, \ldots, y_{k}=x_{j 1}$ be a sequence of vertices of such path in $C$ where $k \geq 2$ and $\left\{y_{i}, y_{i+1}\right\} \in E(C)$ for $i=0,1, \ldots, k-1$. If $k>2$, then $\left\{w, x_{j 1}\right\}$ must be a chord of $\widetilde{C}$, a contradiction. If $k=2$, then $y_{1}=x_{j 2}$ and $C \backslash\left\{y_{1}\right\}$ is also a cycle corresponding to $\widetilde{C}$. This contradicts to the minimality of $C$.

\section{Some Algebraic Properties}

In this section, we investigate algebraic properties of the edge ideal of a graph with a dominating induced matching.

In [10, it is proved that a Cameron-Walker graph is vertex decomposable, in particular, it is sequentially Cohen-Macaulay. But there is a graph with a dominating induced matching which is not sequentially Cohen-Macaulay; the 6-cycle is such an example; see 7, Proposition 4.1].

We obtain some class of vertex decomposable graphs among graphs with a dominating induced matching.

Recall that a graph $G$ on $V$ is called vertex decomposable (see [21, Lemma 4]) if $G$ is an edgeless graph or there exists $v \in V$ with the following 2 properties:

(VD1) $G \backslash v$ and $G \backslash N[v]$ are vertex decomposable; 
(VD2) no independent set in $G \backslash N[v]$ is a maximal independent set in $G \backslash v$.

We call $v \in V$ a shedding vertex of $G$ if $v$ satisfies (VD2). Note that for a vertex $v \in V$, if there exists $w \in V$ such that $N[w] \subset N[v]$, then $v$ is a shedding vertex ([21, Lemma 6]).

Theorem 3.1. Let $G$ be a finite simple graph on $V$ with a dominating induced matching. Assume that there exists a decomposition $V=W \sqcup M$ satisfying the following property, where $W=\left\{y_{1}, \ldots, y_{r}\right\}$ is an independent set and $G_{M}$ consists of $m$ disconnected edges $\left\{x_{j 1}, x_{j 2}\right\}, j=1, \ldots, m$. Moreover assume that for each $j=1, \ldots, m$, one of the following is satisfied:

(i) $\operatorname{deg}_{G} x_{j 1}=1$ or $\operatorname{deg}_{G} x_{j 2}=1$;

(ii) $\operatorname{deg}_{G} x_{j 1}=\operatorname{deg}_{G} x_{j 2}=2$ and there is $y_{i_{j}} \in W$ such that $x_{j 1}, x_{j 2} \in N_{G}\left(y_{i_{j}}\right)$;

(iii) $\operatorname{deg}_{G} x_{j k}=3$ and $\operatorname{deg}_{G} x_{j l}=2$ where $\{k, l\}=\{1,2\}$, and there is $y_{i_{j}} \in W$ such that $N_{G}\left(y_{i_{j}}\right)=\left\{x_{j 1}, x_{j 2}\right\}$;

(iv) $\operatorname{deg}_{G} x_{j 1}=\operatorname{deg}_{G} x_{j 2}=3$ and there are distinct three vertices $y_{i_{j 1}}, y_{i_{j 2}}, y_{i_{j 3}} \in$ $W$ such that $\left\{x_{j 1}, y_{i_{j 1}}\right\},\left\{x_{j 2}, y_{i_{j 2}}\right\} \in E(G), N_{G}\left(y_{i_{j 3}}\right)=\left\{x_{j 1}, x_{j 2}\right\}$, and there is a pendant triangle attached to at least one of $y_{i_{j 1}}, y_{i_{j 2}}$.

Then $G$ is vertex decomposable.

Remark 3.2. The each condition (ii), (iii), (iv) of Theorem 3.1 is concerned with the existence of a pendant triangle.

Indeed, the condition (ii) means that $G$ has a pendant triangle attached to $y_{i_{j}}$; the condition (iii) means that $G$ has a pendant triangle attached to $x_{j k}$; the condition (iv) is explicit.

In order to prove Theorem 3.1, we use the following lemma.

Lemma 3.3. Let $G$ be a finite simple graph on $V$. Suppose that $G$ has a pendant triangle attached to $v \in V$. Then $v$ is a shedding vertex.

Proof. Let $v_{1}, v_{2}$ be the two degree 2 vertices of a pendant triangle attached to $v$. Then $N\left[v_{1}\right]=\left\{v, v_{1}, v_{2}\right\} \subset N[v]$ holds. Hence $v$ is a shedding vertex; see before Theorem 3.1

Now we prove Theorem 3.1 .

Proof of Theorem 3.1. We use induction on $r=\# W$. If $r=1$, then $G$ is chordal and thus $G$ is vertex decomposable by Woodroofe [21, Corollary 7].

Suppose that $r \geq 2$. If the cases (iii) and (iv) do not occur, then $G$ is a CameronWalker graph and thus, $G$ is vertex decomposable by [10, Theorem 3.1].

If there is an edge $\left\{x_{j 1}, x_{j 2}\right\}$ with the condition (iii), say, $\operatorname{deg} x_{j 1}=3$ and $\operatorname{deg} x_{j 2}=2$, then $x_{j 1}$ is a shedding vertex because of Remark 3.2 and Lemma 3.3. Therefore we only need to prove that both $G \backslash x_{j 1}$ and $G \backslash N\left[x_{j 1}\right]$ are vertex decomposable. Indeed $G \backslash x_{j 1}$ is the disjoint union of single edge $\left\{x_{j 2}, y_{i_{j}}\right\}$ and $G^{\prime}:=G \backslash\left\{x_{j 1}, x_{j 2}, y_{i_{j}}\right\}$. Since the vertex set of $G^{\prime}$ can be decomposed as $W^{\prime} \sqcup M^{\prime}$ where $W^{\prime}=W \backslash\left\{y_{i_{j}}\right\}$ and $M^{\prime}=M \backslash\left\{x_{j 1}, x_{j 2}\right\}, G^{\prime}$ has a dominating induced matching. Also $G^{\prime}$ satisfies the assumption of the theorem with this decomposition of the vertex set since $N_{G}\left(y_{i_{j}}\right)=\left\{x_{j 1}, x_{j 2}\right\}$. Hence we conclude that $G^{\prime}$, and thus $G \backslash x_{j 1}$ is vertex decomposable by inductive hypothesis. Also the vertex set of $G \backslash N\left[x_{j 1}\right]$ can be decomposed as $W^{\prime \prime} \sqcup M^{\prime \prime}$ where $W^{\prime \prime}=W \backslash N\left(x_{j 1}\right)$ and $M^{\prime \prime}=M \backslash\left\{x_{j 1}, x_{j 2}\right\}$. Thus $G \backslash N\left[x_{j 1}\right]$ has a dominating induced matching. Since $\operatorname{deg}_{G} x_{j^{\prime} k} \geq \operatorname{deg}_{G \backslash N\left[x_{j 1}\right]} x_{j^{\prime} k}$, we can easily see that this decomposition satisfies 
the assumption of the theorem. Hence by inductive hypothesis, we conclude that $G \backslash N\left[x_{j 1}\right]$ is also vertex decomposable.

Suppose that there is an edge $\left\{x_{j 1}, x_{j 2}\right\}$ with the condition (iv). We may assume that $G$ has a pendant triangle attached to $y_{i_{j 1}}$. Then $y_{i_{j 1}}$ is a shedding vertex by Lemma 3.3. Hence it is enough to prove that both $G \backslash y_{i_{j 1}}$ and $G \backslash N\left[y_{i_{j 1}}\right]$ are vertex decomposable. We first consider $G \backslash y_{i_{j 1}}$. Since the vertex set of this graph can be decomposed as $\left(W \backslash\left\{y_{i_{11}}\right\}\right) \sqcup M$, this graph has a dominating induced matching. We check that each $j^{\prime}=1, \ldots, m$ satisfies one of the conditions (i), (ii), (iii), (iv) with respect to $G \backslash y_{i_{j 1}}$. If $j^{\prime}$ satisfies the condition (i) (resp. (ii)) with respect to $G$, then $j^{\prime}$ satisfies the condition (i) (resp. (ii) or (i)) with respect to $G \backslash y_{i_{j 1}}$. Assume that $j^{\prime}$ satisfies the condition (iii) (resp. (iv)) with respect to $G$. Since $\operatorname{deg}_{G} y_{i_{j 1}} \geq 3$ and $\operatorname{deg}_{G} y_{i_{j^{\prime}}}=2$ (resp. $\operatorname{deg}_{G} y_{i_{j^{\prime} 3}}=2$ ), the vertex $y_{i_{j 1}}$ is different from $y_{i_{j^{\prime}}}$ (resp. $y_{i_{j^{\prime}} 3}$ ). Hence $j^{\prime}$ satisfies the condition (iii) or (ii) (resp. (iv) or (iii)). Therefore this decomposition satisfies the assumption of the theorem. Hence by inductive hypothesis, we conclude that $G \backslash y_{i_{j 1}}$ is vertex decomposable. We next consider $G \backslash N\left[y_{i_{j 1}}\right]$. In this case, the vertex set of $G \backslash N\left[y_{i_{j 1}}\right]$ is decomposed as $W^{\prime} \sqcup M^{\prime}$ where

$$
\begin{aligned}
& W^{\prime}=W \backslash\left(\left\{y_{i_{1}}\right\} \cup \bigcup_{\substack{j^{\prime} \text { satisfying (iii) } \\
\left\{x_{j^{\prime} 1}, x_{j^{\prime} 2}\right\} \cap N_{G}\left(y_{i_{j 1}}\right) \neq \emptyset}}\left\{y_{i_{j^{\prime}}}\right\} \cup \bigcup_{\substack{j^{\prime} \text { satisfying (iv) } \\
\#\left(\left\{x_{j^{\prime} 1}, x_{j^{\prime}}\right\} \cap N_{G}\left(y_{i_{j}}\right)\right)=1}}\left\{y_{i_{j^{\prime}} 3}\right\}\right),
\end{aligned}
$$

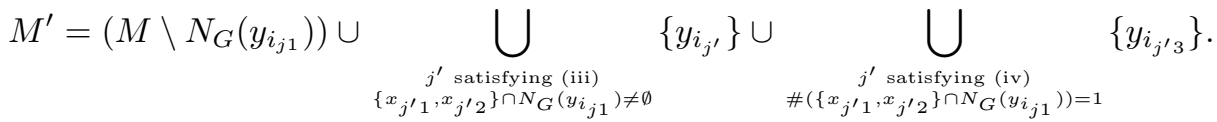

Then we can easily see that $G \backslash N_{G}\left[y_{i_{j 1}}\right]$ has a dominating induced matching. For example, let $j^{\prime}$ be an index satisfying (iii) and $\left\{x_{j^{\prime} 1}, x_{j^{\prime} 2}\right\} \cap N_{G}\left(y_{i_{j 1}}\right) \neq \emptyset$, say $x_{j^{\prime} 1} \in N_{G}\left(y_{i_{j 1}}\right)$. Then $x_{j^{\prime} 1} \notin M^{\prime}$ but $y_{i_{j^{\prime}}} \in M$ and $\left\{x_{j^{\prime} 2}, y_{i_{j^{\prime}}}\right\}$ is an edge of $G \backslash N\left[y_{i_{j 1}}\right]$. Note that in this case, $\operatorname{deg}_{G \backslash N\left[y_{\left.i_{j}\right]}\right]} y_{i_{j^{\prime}}}=1$. Then it is also easy to see that the assumption of the theorem is satisfied with this decomposition. Therefore $G \backslash N\left[y_{i_{j 1}}\right]$ is vertex decomposable by inductive hypothesis.

Although we provide the characterization for a graph with a dominating induced matching to be unmixed in Theorem 2.4, we can obtain a clearer characterization for the unmixedness of the class of graphs in Theorem 3.1. It is sufficient to consider a connected graph which is not a single edge.

Theorem 3.4. Let $G$ be a finite simple connected graph on $V$ with a dominating induced matching. Assume that there exists a decomposition $V=W \sqcup M$ satisfying the assumption of Theorem 3.1 (where $W \neq \emptyset$ ). We use the same notation as in Theorem 3.1 and before Theorem 2.4. Then $G$ is Cohen-Macaulay if and only if $\# W=m_{2}$ and for all $y_{i} \in W$, there is just one edge $\left\{x_{j_{i} 1}, x_{j_{i} 2}\right\}$ of $G$ such that both $\left\{x_{j_{i} 1}, y_{i}\right\}$ and $\left\{x_{j_{i} 2}, y_{i}\right\}$ are edges of $G$.

Proof. We first note that $G$ is Cohen-Macaulay if and only if $G$ is unmixed because $G$ is vertex decomposable by Theorem 3.1 .

("Only If") Let $M_{2}$ be the union of the following subsets $V_{1}, \ldots, V_{4}$ of $M: V_{1}$ is the set of the vertices $x_{j k} \in V$ where $\left\{x_{j 1}, x_{j 2}\right\}$ is an edge of type (i) of Theorem 3.1 with $\operatorname{deg} x_{j k} \geq \operatorname{deg} x_{j l}=1(\{k, l\}=\{1,2\}) ; V_{2}$ is the set of the vertices $x_{j 1}$ where $\left\{x_{j 1}, x_{j 2}\right\}$ is an edge of type (ii) of Theorem 3.1 , $V_{3}$ is the set of the vertices 
$x_{j k}$ where $\left\{x_{j 1}, x_{j 2}\right\}$ is an edge of type (iii) of Theorem 3.1 with $\operatorname{deg} x_{j k}=3$ and $\operatorname{deg} x_{j l}=2(\{k, l\}=\{1,2\}) ; V_{4}$ is the set of the vertices $x_{j k}$ where $\left\{x_{j 1}, x_{j 2}\right\}$ is an edge of type (iv) of Theorem 3.1 and the numbers of pendant triangles attached to each $y_{i_{j} k}$ is less than or equal to that of to $y_{i_{j} l}$ with the notation in Theorem 3.1 (iv) $(\{k, l\}=\{1,2\})$. Clearly, $M_{2}$ satisfies the condition $(* 1)$. The condition $(* 2)$ is also satisfied because $G$ is connected. Note that $\# M_{2}=m$, in particular $m_{2}^{\prime}=0$. Since $G$ is unmixed, by (b1) of Theorem 2.4 it follows that

$$
m_{2}=\# N_{G}\left(M_{2}\right)-\# M_{2} .
$$

Also $W \subset N_{G}\left(M_{2}\right)$ holds. Actually, take $y_{i} \in W$. Since $G$ is connected, there exists an edge $\left\{x_{j 1}, x_{j 2}\right\}$ such that $\left\{x_{j k}, y_{i}\right\}$ is an edge of $G$. If $\left\{x_{j 1}, x_{j 2}\right\}$ is of type (i) or (ii) of Theorem 3.1] then it is easy to see that $y_{i} \in N_{G}\left(M_{2}\right)$. If $\left\{x_{j 1}, x_{j 2}\right\}$ is of type (iii) of Theorem 3.1 and $x_{j k} \notin M_{2}$, then $x_{j \ell} \in M_{2}$ and $\operatorname{deg}_{G} x_{j k}=2$. It then follows that $y_{i} \in N_{G}\left(x_{j \ell}\right) \subset N_{G}\left(M_{2}\right)$. If $\left\{x_{j 1}, x_{j 2}\right\}$ is of type (iv) of Theorem 3.1 and $x_{j k} \notin M_{2}$, then there is a pendant triangle attached to $y_{i}$. Let $x_{j^{\prime} 1}, x_{j^{\prime} 2}$ be the two vertices of the pendant triangle of degree 2 . Since $\left\{x_{j^{\prime} 1}, x_{j^{\prime} 2}\right\}$ is of type (ii) of Theorem 3.1, it follows that $y_{i} \in N_{G}\left(M_{2}\right)$.

The inclusion $W \subset N_{G}\left(M_{2}\right)$ implies that $N_{G}\left(M_{2}\right)=V(G) \backslash M_{2}$. Therefore

$$
\begin{aligned}
m_{2} & =\# N_{G}\left(M_{2}\right)-\# M_{2} \\
& =\left(\# V(G)-\# M_{2}\right)-\# M_{2} \\
& =\# V(G)-2 \# M_{2} \\
& =(2 m+\# W)-2 m \\
& =\# W .
\end{aligned}
$$

Suppose that there exists $y_{i} \in W$ such that $\left\{x_{j 1}, y_{i}\right\},\left\{x_{j 2}, y_{i}\right\},\left\{x_{j^{\prime} 1}, y_{i}\right\},\left\{x_{j^{\prime} 2}, y_{i}\right\} \in$ $E(G)$ for $j \neq j^{\prime}$. It then follows that both $\left\{x_{j 1}, x_{j 2}\right\}$ and $\left\{x_{j^{\prime} 1}, x_{j^{\prime} 2}\right\}$ are of type (ii) of Theorem 3.1 In particular, both $\left\{y_{i}, x_{j 1}, x_{j 2}\right\}$ and $\left\{y_{i}, x_{j^{\prime} 1}, x_{j^{\prime} 2}\right\}$ form pendant triangles attached to $y_{i}$. Assume that there are $\alpha \geq 2$ pendant triangles attached to $y_{i}$; set the two degree 2 vertices of each pendant triangle as $\left\{x_{j_{k} 1}, x_{j_{k}} 2\right\}$, $k=1,2, \ldots, \alpha$. Put $M_{2}^{\prime \prime}=\left\{x_{j_{1} 1}, x_{j_{2} 1}, \ldots, x_{j_{\alpha} 1}\right\}$. Then $M_{2}^{\prime \prime}$ satisfies the condition $(* 1)$ and $(* 2)$. Note that $N_{G}\left(M_{2}^{\prime \prime}\right)=\left\{y_{i}, x_{j_{1} 2}, x_{j_{2} 2}, \ldots, x_{j_{\alpha}}\right\}$ and $m_{2}-m_{2}^{\prime \prime}=\alpha$. Therefore by (b1) of Theorem 2.4, we have

$$
\alpha=m_{2}-m_{2}^{\prime \prime}=\# N_{G}\left(M_{2}^{\prime \prime}\right)-\# M_{2}^{\prime \prime}=(\alpha+1)-\alpha=1,
$$

this contradict to $\alpha \geq 2$. Since $m_{2}=\# W$, the assertion follows.

("If") Let $X$ be a maximal independent set of $G$. In order to prove that $G$ is unmixed, it is sufficient to show that $\# X=m$. Set

$$
\begin{aligned}
& E_{1}:=\left\{\left\{x_{j 1}, x_{j 2}\right\} \in E(G): \operatorname{deg}_{G} x_{j 1}=1 \text { or } \operatorname{deg}_{G} x_{j 2}=1\right\}, \\
& E_{2}:=\left\{\left\{x_{j 1}, x_{j 2}\right\} \in E(G): \operatorname{deg}_{G} x_{j 1} \geq 2 \text { and } \operatorname{deg}_{G} x_{j 2} \geq 2\right\}
\end{aligned}
$$

and $V_{k}:=\bigcup_{e \in E_{k}} e$ for $k=1,2$. Since $V=V_{1} \sqcup V_{2} \sqcup W$,

$$
\# X=\#\left(X \cap V_{1}\right)+\#\left(X \cap\left(V_{2} \cup W\right)\right) \text {. }
$$

Take $\left\{x_{j 1}, x_{j 2}\right\} \in E_{1}$. Assume that $\operatorname{deg}_{G} x_{j 1}=1$. Then $\operatorname{deg}_{G} x_{j 2} \geq 2$ because $G$ is connected. If $x_{j 2} \notin X$, then $x_{j 1} \in X$ because the maximality of $X$. This implies $\#\left(X \cap\left\{x_{j 1}, x_{j 2}\right\}\right)=1$ and $\#\left(X \cap V_{1}\right)=m_{1}$.

By assumption, $V_{2} \sqcup W$ can be decomposed as $\bigcup_{i=1}^{r}\left\{y_{i}, x_{j_{i} 1}, x_{j_{i} 2}\right\}$. We claim that $X \cap\left\{y_{i}, x_{j_{i} 1}, x_{j_{i} 2}\right\}=1$ for each $i$. 
Since $\left\{y_{i}, x_{j_{i} 1}\right\},\left\{y_{i}, x_{j_{i} 2}\right\},\left\{x_{j_{i} 1}, x_{j_{i}}\right\} \in E(G)$, it follows that $\#\left(X \cap\left\{y_{i}, x_{j_{i} 1}, x_{j_{i} 2}\right\}\right) \leq$ 1. Assume that $y_{i} \notin X$. If $\operatorname{deg} y_{i} \geq 3$, then $\operatorname{deg}_{G} x_{j_{i} 1}=\operatorname{deg}_{G} x_{j_{i} 2}=2$ and it follows from the maximality of $X$ that exactly one of $x_{j_{i} 1}, x_{j_{i}}$ belongs to $X$. When $\operatorname{deg} y_{i}=2$, if neither $x_{j_{i} 1}$ nor $x_{j_{i} 2}$ belong to $X$, then $X \cup\left\{y_{i}\right\}$ is also an independent set. This contradicts to the maximality of $X$. Thus (exactly) one of $x_{j_{i} 1}, x_{j_{i} 2}$ belongs to $X$. Hence $\#\left(X \cap\left(V_{2} \cup W\right)\right)=m_{2}=\# W$.

Therefore

$$
\# X=\#\left(X \cap V_{1}\right)+\#\left(X \cap\left(V_{2} \cup W\right)\right)=m_{1}+m_{2}=m,
$$

as desired.

We show an example satisfying the assumption of Theorem 3.1 .

Example 3.5. The graph $G$ in Figure 3 has a dominating induced matching, which is not a Cameron-Walker graph. It also satisfies the assumption of Theorem 3.1 with the displayed decomposition of the vertex set.

Then $m_{2}=\# W=3$. We can also easily see that this graph satisfies the assumption for the vertex in $W$ of Theorem 3.4. Hence $G$ is Cohen-Macaulay by Theorem 3.4 .

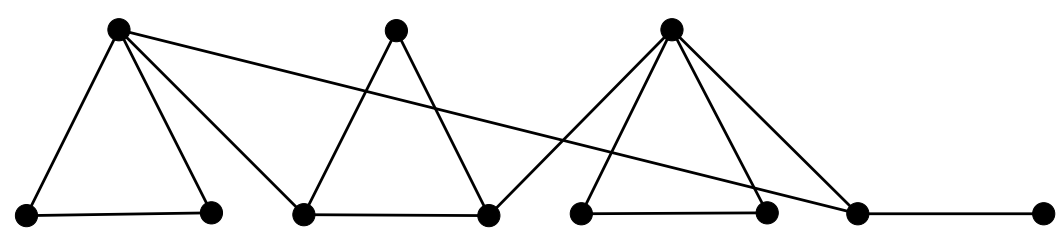

FIGURE 3. A Cohen-Macaulay graph with a dominating induced matching

We close the paper by giving some more examples of a graph with a dominating induced matching which does not satisfy the assumption on Theorem 3.1 .

We first show some Cohen-Macaulay graphs with a dominating induced matching.

Example 3.6. (1) The path graph $P_{4}$ with 4 vertices is a Cohen-Macaulay graph. Also it has a dominating induced matching. Indeed, set $V\left(P_{4}\right)=$ $\{1,2,3,4\}$ and $E\left(P_{4}\right)=\{\{1,2\},\{2,3\},\{3,4\}\}$. Then we see that $P_{4}$ has a dominating induced matching with the decomposition $V\left(P_{4}\right)=W \sqcup M$ where $W=\{1,4\}$ and $M=\{2,3\}$.

(2) The graph $G_{1}$ on the vertex set $\{1,2,3,4,5,6\}$ whose edge set is

$$
E\left(G_{1}\right)=\{\{1,3\},\{1,4\},\{1,5\},\{2,4\},\{2,5\},\{2,6\},\{3,4\},\{5,6\}\}
$$


is a graph with a dominating induced matching. This is Cohen-Macaulay, in particular, unmixed.

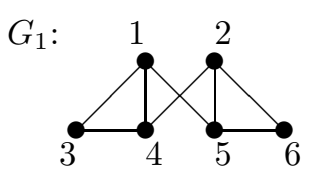

We next show an unmixed graph with a dominating induced matching but not Cohen-Macaulay.

Example 3.7. The graph $G_{2}$ on the vertex set $\{1,2,3,4,5,6\}$ whose edge set is

$$
\{\{1,3\},\{1,4\},\{1,5\},\{1,6\},\{2,3\},\{2,4\},\{2,5\},\{2,6\},\{3,4\},\{5,6\}\}
$$

is a graph with a dominating induced matching. This is unmixed but not CohenMacaulay.

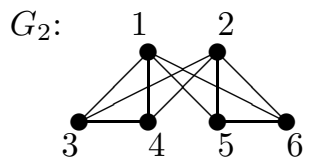

Finally, we show a sequentially Cohen-Macaulay graph with a dominating induced matching but not unmixed.

Example 3.8. The graph $G_{3}$ on the vertex set $\{1,2,3,4,5,6\}$ whose edge set is

$$
\{\{1,3\},\{3,4\},\{2,4\},\{2,5\},\{2,6\},\{5,6\}\}
$$

is a graph with a dominating induced matching.

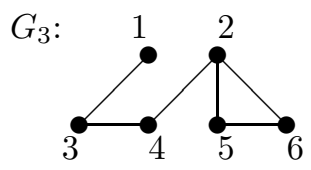

This is not unmixed since both $\{3,4,5,6\}$ and $\{2,3,6\}$ are minimal vertex covers of $G_{3}$. Also, since $G_{3}$ is chordal, it is sequentially Cohen-Macaulay by Francisco and Van Tuyl [7.

Acknowledgment. The third author is partially supported by JSPS Grant-in-Aid for Young Scientists (B) 24740008.

We thank anonymous referees for reading the manuscript carefully.

\section{REFERENCES}

[1] T. Biyikoğlu and Y. Civan, Vertex decomposable graphs, codismantlability, CohenMacaulayness and Castelnuovo-Mumford regularity, Electron. J. Combin. 21 (2014), 17pp.

[2] T. Biyikoğlu and Y. Civan, Bounding Castelnuovo-Mumford regularity of graphs via Lozin's transformation, preprint, arXiv:1302.3064 1 .

[3] T. Biyikoğlu and Y. Civan, Castelnuovo-Mumford regularity of graphs, preprint, arXiv:1503.06018

[4] K. Cameron and T. Walker, The graphs with maximum induced matching and maximum matching the same size, Discrete Math. 299 (2005), 49-55.

[5] D. M. Cardoso, E. A. Martins, L. Media, and O. Rojo, Spectral results for the dominating induced matching problem, preprint, arXiv:1311.2748v1. 
[6] H. Dao, C. Huneke and J. Schweig, Bounds on the regularity and projective dimension of ideals associated to graphs, J. Algebraic Combin. 38 (2013), 37-55.

[7] C. A. Francisco and A. Van Tuyl, Sequentially Cohen-Macaulay edge ideals, Proc. Amer. Math. Soc. 135 (2007), 2327-2337.

[8] I. Gitler and C. E. Valencia, Bounds for invariants of edge-rings, Comm. Algebra 33 (2005), 1603-1616.

[9] H. T. Hà and A. Van Tuyl, Monomial ideals, edge ideals of hypergraphs, and their graded Betti numbers, J. Algebraic Combin. 27 (2008), 215-245

[10] T. Hibi, A. Higashitani, K. Kimura, and A. B. O'Keefe, Algebraic study on Cameron-Walker graphs, J. Algebra 422 (2015), 257-269.

[11] G. Kalai and R. Meshulam, Intersections of Leray complexes and regularity of monomial ideals, J. Combin. Theory Ser. A 113 (2006), 1586-1592.

[12] M. Katzman, Characteristic-independence of Betti numbers of graph ideals, J. Combin. Theory, Ser. A 113 (2006), 435-454.

[13] F. Khosh-Ahang and S. Moradi, Regularity and projective dimension of edge ideal of $C_{5}$-free vertex decomposable graphs, Proc. Amer. Math. Soc. 142 (2014), 1567-1576.

[14] K. Kimura, Non-vanishingness of Betti numbers of edge ideals, in: Harmony of Gröbner bases and the modern industrial society, World Scientific, 2012, pp. 153-168.

[15] M. Kummini, Regularity, depth and arithmetic rank of bipartite edge ideals, J. Algebraic Combin. 30 (2009), 429-445.

[16] M. C. Lin, M. J. Mizrahi, J. L. Szwarcfiter, Exact algorithms for dominating induced matchings, preprint, arXiv:1301.7602 2 .

[17] G. Lyubeznik, A new explicit finite free resolution of ideals generated by monomials in an R-sequence, J. Pure Appl. Algebra 51 (1988), 193-195.

[18] M. Mahmoudi, A. Mousivand, M. Crupi, G. Rinaldo, N. Terai, and S. Yassemi, Vertex decomposability and regularity of very well-covered graphs, J. Pure Appl. Algebra 215 (2011), $2473-2480$.

[19] E. Nevo, Regularity of edge ideals of $C_{4}$-free graphs via the topology of the lcm-lattice, $J$. Combin. Theory, Ser. A 118 (2011), 491-501.

[20] A. Van Tuyl, Sequentially Cohen-Macaulay bipartite graphs: vertex decomposability and regularity, Arch. Math. (Basel) 93 (2009), 451-459.

[21] R. Woodroofe, Vertex decomposable graphs and obstructions to shellability, Proc. Amer. Math. Soc. 137 (2009), 3235-3246.

[22] R. Woodroofe, Matchings, coverings, and Castelnuovo-Mumford regularity, J. Commut. Algebra 6 (2014), 287-304.

[23] X. Zheng, Resolutions of facet ideals, Comm. Algebra 32 (2004), 2301-2324.

(Takayuki Hibi) Department of Pure and Applied Mathematics, Graduate School of Information Science and Technology, Osaka University, Toyonaka, Osaka 560-0043, JAPAN

E-mail address: hibi@math.sci.osaka-u.ac.jp

(Akihiro Higashitani) Department of Mathematics, Kyoto Sangyo University, Motoyama, Kamigamo, Kita-KU, KYOtO 603-8555, JaPAN

E-mail address: ahigashi@cc.kyoto-su.ac.jp

(Kyouko Kimura) Department of Mathematics, Faculty of Science, Shizuoka UniverSity, 836 Ohya, Suruga-Ku, Shizuoka 422-8529, JAPAN

E-mail address: kimura.kyoko.a@shizuoka.ac.jp

(Akiyoshi Tsuchiya) Department of Pure and Applied Mathematics, Graduate School of Information Science and Technology, Osaka University, Toyonaka, Osaka 560-0043, JAPAN

E-mail address: a-tsuchiya@cr.math.sci.osaka-u.ac.jp 